\title{
Understanding the WMAP Cold Spot mystery ${ }^{*}$.
}

\author{
P. D. Naselsky,,$^{1, * *}$ P. R. Christensen, ${ }^{1,{ }^{* * *}}$ P. Coles,${ }^{2,}{ }^{* * * *}$ \\ O. V. Verkhodanov, ${ }^{3,{ }^{* * * * *}}$ D. I. Novikov, ${ }^{4,{ }^{* * * * * *}}$ and Jai. Kim ${ }^{1,{ }^{* * * * * * *}}$ \\ ${ }^{1}$ Niels Bohr Institute, Blegdamsvej 17, DK-2100 Copenhagen, Denmark \\ ${ }^{2}$ School of Physics \& Astronomy, Cardiff University, 5 \\ The Parade, Cardiff, CF24 3AA, Wales, United Kingdom \\ ${ }^{3}$ Special Astrophysical Observatory, Nizhnij Arkhyz, Karachaj-Cherkesia, 369167, Russia \\ ${ }^{4}$ Imperial College, London, United Kingdom
}

The first and third year data releases from the Wilkinson Microwave Anisotropy Probe (WMAP) provide evidence of an anomalous Cold Spot (CS) at galactic latitude $b=-57^{\circ}$ and longitude $l=209^{\circ}$. We have examined the properties of the CS in some detail in order to assess its cosmological significance. We have performed a cluster analysis of the local extrema in the CMB signal to show that the CS is actually associated with a large group of extrema rather than just one. We have also checked the idea that the CMB signal has a non-Gaussian tail. For each ring we apply a linear filter with characteristic scale $R$, dividing the CMB signal in two parts: the filtered part, with characteristic scale above that of the filter $R$, and the difference between the initial and filtered signal. Using the filter scale as a variable, we can maximize the skewness and kurtosis of the smoothed signal and minimize these statistics for the difference between initial and filtered signal. We find that, unlike its Northern counterpart, the Southern Galactic hemisphere of the CMB map is characterized by significant departure from Gaussianity of which the CS is not the only manifestation: we have located a ring, on which there are "cold" as "hot" spots with almost the same properties as the CS. Exploiting the similarity of the WCM and the ILC maps, and using the latter as a guide map, we have discovered that the shape of the CS is formed primarily by the components of the $\mathrm{CMB}$ signal represented by multipoles between $10 \leq \ell \leq 20$, with a corresponding angular scale about $5-10^{\circ}$. This signal leads to modulation of the whole CMB sky, clearly seen at $|b|>30^{\circ}$ in both the 
ILC and WCM maps, rather than a single localized feature. After subtraction of this modulation, the remaining part of the CMB signal appears to be consistent with statistical homogeneity and Gaussianity. We therefore infer that the mystery of the WMAP CS reflects directly the peculiarities of the low-multipole tail of the CMB signal, rather than a single local (isolated) defect or manifestation of a globally anisotropic cosmology.

\section{INTRODUCTION}

An extremely Cold Spot (CS), apparently inconsistent with the assumption of statistically homogeneous Gaussian fluctuations, was detected in a wavelet analysis [1-3] of the first-year data release from the Wilkinson Microwave Anisotropy Probe (WMAP). More recently, the existence of this spot has been confirmed [4, 5] the WMAP third year data release [6, 8]. The WMAP CS is centered at the position $b=-57^{\circ}, l=209^{\circ}$ in Galactic Coordinates and has a characteristic scale about $10^{\circ}$. As was pointed out Cruz et al. [9], the frequency dependence of the signal in the spot area is extremely flat. This fact has been used by the authors mentioned above to argue that the WMAP CS belongs to the CMB signal, rather than any form of foreground emission. Cruz et al. pointed out that the reason the CS was not been detected in real space before the wavelet analysis was that it was hidden amongst structures at different scales.

As an apparent example of non-Gaussian behavior in the WMAP CMB signal, the CS has attracted very serious attention from the theoretical point of view. Tomita [10] suggested that the CS can be related to second-order gravitational effects. Inoue and Silk [11] proposed a model involving local compensated voids. The origin of the CS in connection to the brightness and number of counts of the NVSS [12] sources (smoothed on the scale of a few degrees) was recently discussed by Rudnick, Brown and Williams [11]. They have detected

\footnotetext{
** $\quad$ Electronic address: naselsky@nbi.dk

*** Electronic address: perrex@nbi.dk

${ }^{* * * *}$ Electronic address: peter.coles@astro.cf.ac.uk

***** Electronic address: vo@sao.ru

****** Electronic address: d.novikov@imperial.ac.uk

******* Electronic address: jkim@nbi.dk
} 
a 20-45\% dip in the smoothed NVSS source counts which can be interpreted, they argue, as a manifestation of the integrated Sachs-Wolfe effect, seen for a single region of the CMB sky. Jaffe et al. [14, 15], Cayon et al. [16] and McEwen et al. [17, 18] have investigated the Bianchi $\mathrm{VII}_{h}$ anisotropic cosmological model as a possible explanation of the CS and other features of the WMAP low multipoles. Recently, Cruz et al. [5, 19] have pointed out that the CS could be produced by a cosmic texture, assuming that the CMB signal is a combination of the Gaussian and non-Gaussian parts. The present status of the problem of existence of the CS remains uncertain, despite the presence of a vast collection of theoretical suggestions. Nevertheless, if we believe that one particular part of the WMAP CMB signal contains non-Gaussian features, it would be necessary to seek corroborating evidence of nonGaussianity elsewhere in order to understand their properties more fully. In this Paper we therefore present a detailed investigation of the properties of the CS, focusing attention on the following topics.

First, in Section 2, we show how the CS can be easily detected in the pixels domain not only in the derived CMB signal, but even in the WMAP maps for K-W bands before separation of the signal into CMB and foreground components.

Second, we will demonstrate that the CS belongs to a cluster of local minima, the spatial distribution of which is modulated by the large-angle modes of the CMB signal outside the Galactic plane. For that we use the Internal Linear Combination (ILC) III map and the co-added WCM map with $N_{\text {side }}=512$ in the HEALPix format, converted to GLESP format [20], where each iso-latitude ring has the same number of pixels $N_{\phi}=2048$ in azimuthal direction $\phi$ in polar coordinates. After that we perform a cluster analysis [21] of the positive and negative peaks for selected rings in the area outside the Kp0 mask, mostly concentrating our attention on the ring crossing the CS at its extrema $b=-57^{\circ}$ and $-180^{\circ} \leq \phi \leq 180^{\circ}$. Taking into consideration the signal for each ring with the latitude $b$, we can investigate the morphology of the CMB signal at each latitude for the whole range of $\phi$. This approach allows to connect the morphology of the CS to the signal outside the CS for the same latitude $b=-57^{\circ}$. We will show that the cluster contained the CS, is not a unique feature of the $b=-57^{\circ}$ iso-latitude ring. For example, close to the CS there are two significant clusters of maxima, but these peaks have lower amplitude that the CS.

Next, since the origin of large clusters of extrema is related to the angular modulation of the signal on large scales [21], we split the CMB signal into two parts. To do that we use the 
skewness and the kurtosis of the signal for selected rings, including the $b=-57^{\circ}$ ring. Then by using a simple linear smoothing filter with characteristic scale $R$ we separate the signal into a smoothed component and to a difference between initial signal and the smoothed component. For the smoothed signal we define the skewness $S(R)$ and the kurtosis $K(R)$ as a functions of $R$ and find the scale of filtering which maximize both these characteristics $S\left(R_{\text {opt }}\right), K\left(R_{\text {opt }}\right) \rightarrow \max$. By using this scale $R_{\max }$ we separate the initial CMB signal in two parts, one of them (the smoothed one) contain a maximally non-Gaussian signal, and the another one (initial signal minus the smoothed one) the maximally Gaussian signal. The non-Gaussian part is mainly formed by the signal localized at the range of multipoles $2 \leq \ell \leq 20$ and the other one belongs to the $\ell>20$ multipoles. Our analysis clearly demonstrates that the pronounced non-Gaussianity of the CS reflects directly the existence of a large-scale angular modulation of the CMB signal with $10 \leq \ell \leq 20$.

Finally, using cluster analysis in combination with skewness and kurtosis statistics, we are able to detect a few additional cold and hot spots on the same $b=-57^{\circ}$ ring as the famous one. To show that the effect on clustering of the peak by low multipoles of the CMB is very common, we took into consideration the north Galactic hemisphere with deficit of the power and have found a few cold and hot spots. The idea of implementing of cluster analysis in combination with skewness and kurtosis statistics was stimulated in [1-4, 9], and especially in [22] and [23].

In our analysis we use both the WCM map and the ILC third year map, which are very similar outside the $b= \pm 25^{\circ}$ cut of the Galactic plane. For our analysis we use the high resolution ILC III map as a guide map to mark possible zones of the CMB sky in which the enhanced clustering of the peaks is expected to be considerable.

\section{2. "NAIVE" DETECTION OF THE WMAP COLD SPOT}

As was pointed out in the Introduction, historically the CS was detected in the WMAP data as one of the deepest minima of the CMB signal, using method based on wavelets. Our first aim is to show that this CS can actually be detected quite straightforwardly in the pixel domain using simple threshold techniques. In Fig.1 we take two thresholds of the ILC III signal at the range of temperatures $-0.2 \leq \Delta T \leq-0.05$ and $0.05 \leq \Delta T \leq 0.11 \mathrm{mK}$ and map them with a color scale $-0.2,0$ and $0,0.2$, respectively. 

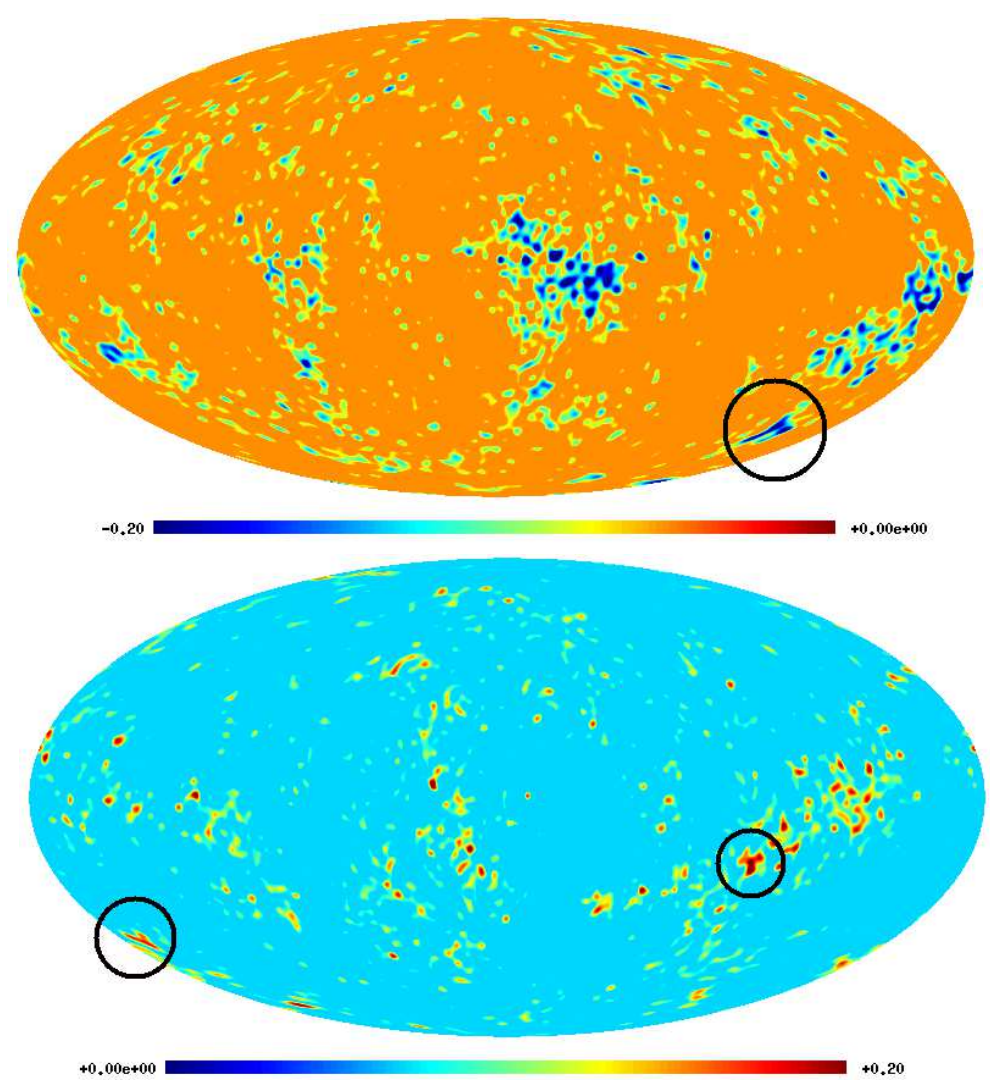

Figure 1. The map for negative (top) and positive (bottom) thresholds $-0.2 \leq T \leq-0.05$ and $0.05 \leq T \leq 0.2$ of the ILC III map. For the top map the black circle marks the location of the CS in the Galactic coordinates. For the bottom map the circles mark high amplitude positive peaks.

From this figure one can immediately see the difference in statistical properties of the positive and negative thresholds of the map. First of all, it is important to note that a majority of the positive peaks are associated with the area around $|b| \leq 25-30^{\circ}$ (see the bottom plot), while the negative peaks are present well outside this region of the map. Secondly, the amplitude of the highest positive peaks is limited by $0.11 \mathrm{mK}$, while for the negative peaks it is about $-0.019 \mathrm{mK}$. For the CS the temperature of the negative peak is in agreement with the estimate [4]. In Fig.2, by using the SKY Viewer, we map the ILC III $\mathrm{K}$ and $\mathrm{Ka}$ maps, in which the CS is located in the center of the area marked by the black circle (the blue cluster of peaks inside the black circle). From the $\mathrm{K}$ and Ka maps one can clearly see that the CS is clearly observed even without subtraction of the CMB signal. In agreement with $[2,13]$ we note that there is a cluster of negative peaks, rather than one single peak.

To show the local structure of the zone containing the CS, in Fig.3 we plot high resolution 

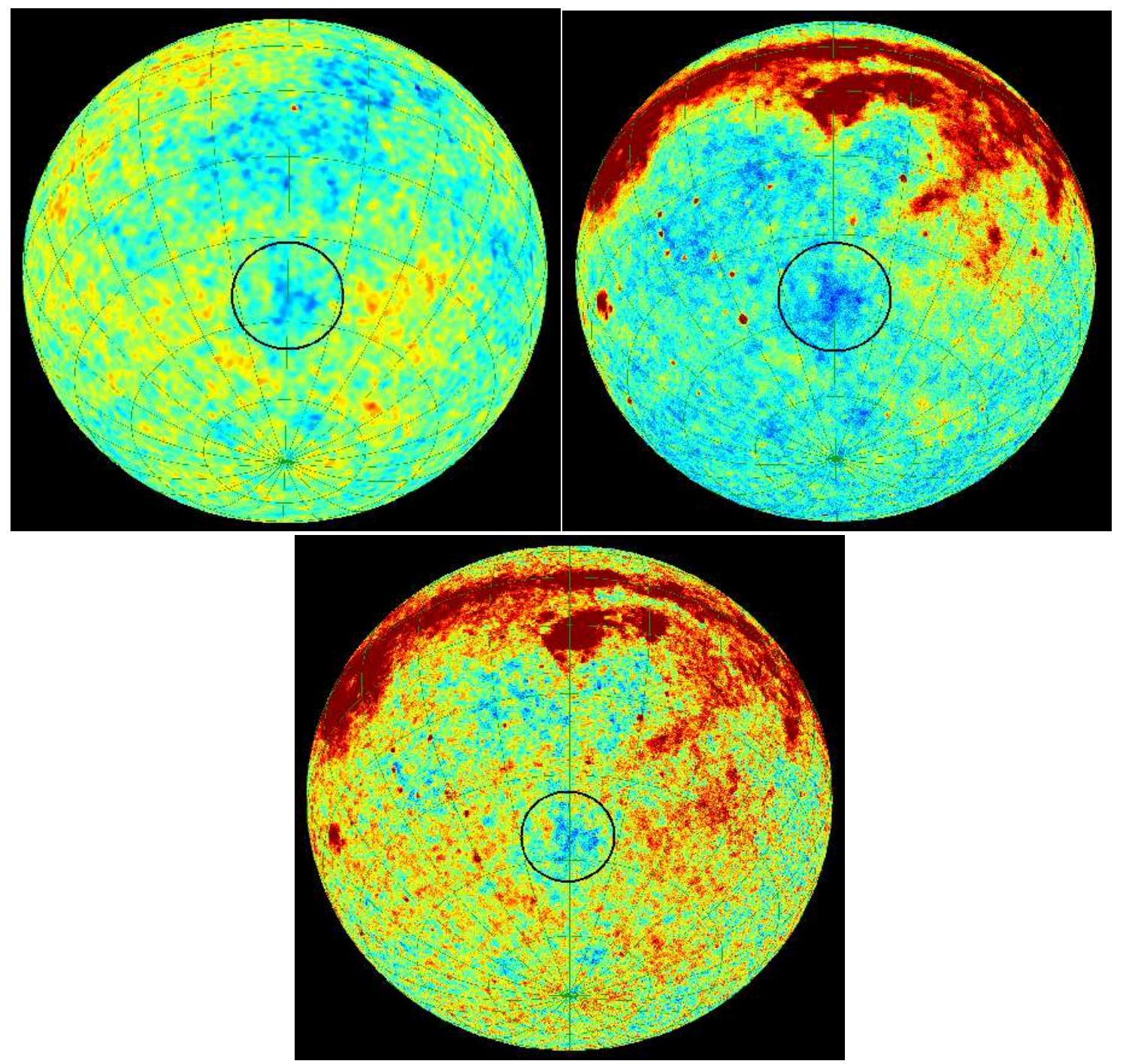

Figure 2. The SKY Viewer maps for the ILC III (top), the K and Ka bands of the WMAP (second and third from the top) map with cutoff of intensity down to the minimal values. For all the maps the zone of the CS has been placed at the center of the black circle.

$\left(\ell_{\max }=100\right)$ images of the inner structure of the CS zone, including the Haslam et al. map [24]. which is expected to be free from CMB "contamination".

From Fig.2 and Fig.3 one can draw the important conclusion that the zone of the CS is surrounded by zones of hot spots, clearly seen in Fig.2 just on the right and left hand side of the CS.

If the origin of the CS is related to large-angular modulation of the CMB map and possible anisotropy of the power distribution across the sky, it would be naturally to expect that additionally to the WMAP CS detected in [1] and others, we could find other cold and 

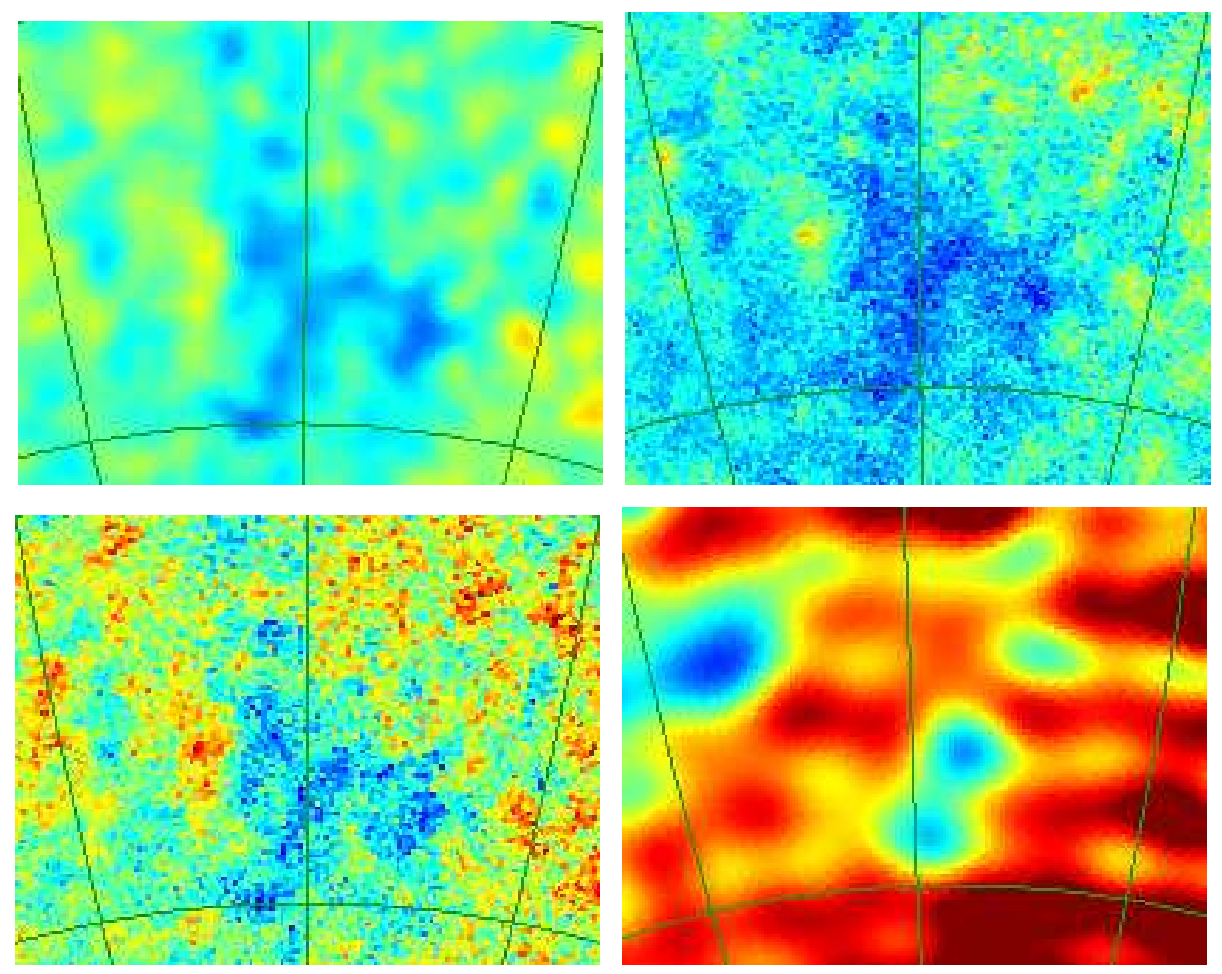

Figure 3. The CS zone image projection (in the pixel domain) of the ILC III, K, Ka and Haslam et al. maps. The size of all the maps is $25 \times 25^{\circ}$.

hot spots similar or even equal morphological properties. To show that this is the case for WCM and the ILC III maps, in the next section we will use a cluster analysis of these maps in order to mark possible zones of peculiar distribution (clusters) in the signal.

\section{CLUSTER ANALYSIS OF THE CMB MAPS IN THE VICINITY OF THE CS}

The main idea behind our implementation of cluster analysis is to divide the CMB sky above and below the Kp0 mask into iso-latitude rings and then to analyze the properties of the signal for each ring separately. First, let us briefly describe the statistical properties of the signal $T\left(\theta=\theta_{c}, \varphi\right)$ for fixed latitude $b$. Let us take under consideration the distribution of peaks above and below some given threshold $\nu_{t}=\Delta T / \sigma_{0}$, where $\sigma_{0}^{2}$ is the variance for each ring. For a one-dimensional cross-section of the CMB map we introduce a definition of a cluster of maxima considering, for example, two points, $x_{1}$ and $x_{2}\left(x_{1}<x_{2}\right)$ from the ring. If for all the points in the interval $x_{1} \leq x \leq x_{2}$ we have $\Delta T(x)>\nu_{t} \sigma_{0}$, we call these points $x_{1}, x_{2}$ connected to each other with respect to the threshold $\nu_{t}$. A collection of maxima of $\Delta T$ located at the points $\left\{x_{k}\right\}, k=1,2 . . K$ we will call a cluster of length $D$ 
if all the points $\left\{x_{k}\right\}$ are connected to each other when the threshold $\nu_{t}$ is applied ${ }^{1}$. The length of the cluster $D=\left|x_{1}-x_{2}\right| / 2 \pi$ is an analogue of the definition of the two-dimensional area [4], but in a one-dimensional case. For a random Gaussian field the statistic of length of the clusters (one-dimensional) and the area (two-dimensional) are similarly sensitive to the spectral parameters of the random field, as described by [25]. However, the difference between cluster analysis and area statistics becomes obvious in application to non-Gaussian random fields. Let us assume for a moment that some zone of the signal is characterized by the area statistic, and that this has a very non-Gaussian value. To characterize the properties of the non-Gaussian field it seems to be very important to know, if this area is related to a uniform structure with one single peak, or formed from a cluster of peaks with the same area. This is why for our analysis of the WCM and the ILC III statistical properties we prefer to use cluster analysis (CA). In our analysis, in addition to the length of cluster $D$, we will use its size $S$ which we define as the number of maxima (or minima) above (or below) the threshold $\nu_{t}$ within the interval length $D=\left|x_{1}-x_{2}\right| / 2 \pi$.

\subsection{Statistical properties of the signals for equal latitude ring}

To describe the statistical properties of equal latitude rings we will use the approach proposed in [26].

The standard treatment for a full-sky CMB signal $T(\theta, \varphi)$ is via spherical harmonic decomposition:

$$
T(\theta, \varphi)=\sum_{\ell=0}^{\ell_{\max }} \sum_{m=-\ell}^{\ell} a_{\ell m} Y_{\ell m}(\theta, \varphi),
$$

where $\theta$ and $\varphi$ are the polar and azimuthal angle, respectively, and $a_{\ell m}$ are the spherical harmonic coefficients. Here $Y_{\ell m}$ are the spherical harmonics, defined in terms of Legendre polynomials and plane waves:

$$
Y_{\ell m}(\theta, \varphi)=N_{\ell m} P_{\ell}^{m}(\cos \theta) \exp (i m \varphi)
$$

where

$$
N_{\ell m}=(-1)^{m} \sqrt{\frac{(2 \ell+1)(\ell-m) !}{4 \pi(\ell+m) !}} .
$$

\footnotetext{
${ }^{1}$ The same definition can be introduced for cluster of minima, when instead of maxima we will use the minima of $T$.
} 
For a random Gaussian CMB sky the properties of the statistical ensemble of realizations are completely specify by the power spectrum

$$
C(\ell)=\frac{1}{2 \ell+1} \sum_{m=-\ell}^{m=\ell}\left\langle\left|a_{\ell, m}\right|^{2}\right\rangle
$$

while for each single realization we expect to find some deviation from $C(\ell)$ due to the "cosmic variance" effect. Below we will use a polar coordinate system in which the Galactic plane $(b=0)$ is associated with $\theta=\pi / 2$.

Let us analyze the signal $T\left(\theta_{c}, \varphi\right)$ from the equal-latitude ring at $\theta=\theta_{c}$, where $\left|\theta_{c}\right| \geq$ $\left|\theta_{\text {mask }}\right|$, where $\left|\theta_{\text {mask }}\right|$ is the maximum latitude of any foreground masks. This $\operatorname{ring} T\left(\theta_{c}, \varphi\right) \equiv$ $T_{c}(\varphi)$ is a one-dimensional signal, for which we can construct a Fourier transform with coefficients $g_{m}$ :

$$
T_{c}(\varphi)=\sum_{m=-\ell_{\max }}^{\ell_{\max }} g_{m} \exp (i m \varphi)
$$

where

$$
g_{m}=\int_{0}^{2 \pi} d \varphi T_{c}(\varphi) \exp (-i m \varphi)
$$

We can then relate the ring to the full-sky signal via Eq.(2) and (5) and get

$$
g_{m}=\sum_{\ell \geq|m|}^{\ell_{\max }} N_{\ell m} P_{\ell}^{m}\left(\cos \theta_{c}\right) a_{\ell m}
$$

That is to say that the Fourier coefficients $g_{m}$ of the ring can be expressed as a combination of the full-sky $a_{\ell m}$. Defining the variance of the signal for equal latitude ring as

$$
\operatorname{Var} T=\frac{1}{2 \pi} \int_{0}^{2 \pi} d \varphi[T(\varphi)-\langle T\rangle]^{2},\langle T\rangle=\frac{1}{2 \pi} \int_{0}^{2 \pi} d \varphi T(\varphi)
$$

and after substitution of Eq.(5) into Eq.(8) we have

$$
\begin{array}{r}
\langle T\rangle=\sum_{\ell} N_{\ell, m=0} P_{\ell}(\cos \theta) a_{\ell, m=0}, \\
\operatorname{Var} T=\sum_{\ell} \sum_{\ell^{\prime}} \sum_{m \neq 0} N_{\ell, m} N_{\ell^{\prime}, m} P_{\ell, m}(\cos \theta) \times \\
\times P_{\ell^{\prime}, m}(\cos \theta) a_{\ell, m} a_{\ell^{\prime}, m}^{*} .
\end{array}
$$

For a random Gaussian field (GRF), after average over realizations, the combinations of the $a_{\ell m}$ coefficients in Eq.(9) satisfy the following conditions:

$$
\left\langle a_{\ell, m} a_{\ell^{\prime}, m^{\prime}}^{*}\right\rangle=C(\ell) \delta_{\ell, \ell^{\prime}} \delta_{m, m^{\prime}}
$$


and then

$$
\operatorname{Var} T=\sum_{\ell} \sum_{m \neq 0} N_{\ell, m}^{2} P_{\ell, m}^{2}(\cos \theta) C(\ell)=\sum_{\ell} W(\ell, \cos \theta) C(l)
$$

where $W(\ell, \cos \theta)=\sum_{m \neq 0} N_{\ell, m}^{2} P_{\ell, m}^{2}(\cos \theta)$ is the window function of the ring.

Our approach here is a special case for a well known theoretical prediction: any $n$ dimensional cross sections of $N$ dimensional Gaussian random signal produce a Gaussian signal as well. However, this general theory tells us about the GRF, represented as a sets of realizations. For one single realization of the CMB sky, as the WMAP signal does, the Eq.(11) is no longer available. More general, instead of Eq.(11) we have

$$
a_{\ell, m} a_{\ell^{\prime}, m^{\prime}}^{*}=C(\ell) G_{\ell, \ell^{\prime}}^{m, m^{\prime}},\left\langle G_{\ell, \ell^{\prime}}^{m, m^{\prime}}\right\rangle=\delta_{\ell, \ell^{\prime}} \delta_{m, m^{\prime}}
$$

Thus,

$$
\begin{array}{r}
\operatorname{Var} T=\sum_{\ell} \sum_{\ell^{\prime}} \sum_{m, m^{\prime} \neq 0} N_{\ell, m} N_{\ell^{\prime}, m^{\prime}} P_{\ell, m}(\cos \theta) \times \\
P_{\ell^{\prime}, m^{\prime}}(\cos \theta) C(l) G_{\ell, \ell^{\prime}}^{m, m^{\prime}}, \\
W(\ell, \cos \theta)=\sum_{\ell^{\prime}} \sum_{m \neq 0} N_{\ell, m} N_{\ell^{\prime}, m^{\prime}} \times \\
P_{\ell, m}(\cos \theta) P_{\ell^{\prime}, m^{\prime}}(\cos \theta) G_{\ell, \ell^{\prime}}^{m, m^{\prime}}
\end{array}
$$

The matrix $G_{\ell, \ell^{\prime}}^{m, m^{\prime}}$ describes the coupling between different modes $\ell, m$ and $\ell^{\prime}, m^{\prime}$ for the random process $T(\varphi)$, which leads to variations of the variance $\operatorname{Var} T(\theta)$ for different rings $(\theta=$ const $)$. One can see that integration of Eq.(13) over $\theta$ preserves spontaneous correlations $\left(G_{\ell, \ell^{\prime}}^{m, m^{\prime}} \neq \delta_{\ell, \ell^{\prime}} \delta_{m, m^{\prime}}\right)$ even for the whole sky. However, for a particular ring we will have an additional modulation of these correlations depending on the $\theta$ through the window function $W(\ell, \cos \theta)$. In Fig.4 we show the dependence of $\operatorname{Var} T$ on the galactic latitude for the WCM and the ILC III maps.

First of all, we stress the remarkable similarity of these maps outside the region of the Kp0 mask. Moreover, the variance of the WCM map per each ring matches the variance of the ILC III almost exactly for all latitudes, except for the $|b| \leq 5^{\circ}$ zone. Secondly, note that the $b=-57^{\circ}$ ring lies near to the local maxima of the variance. The width of this zone is about $\theta \sim 10^{\circ}$. Thirdly, from Fig.4 one can clearly see the asymmetry of the variance for 


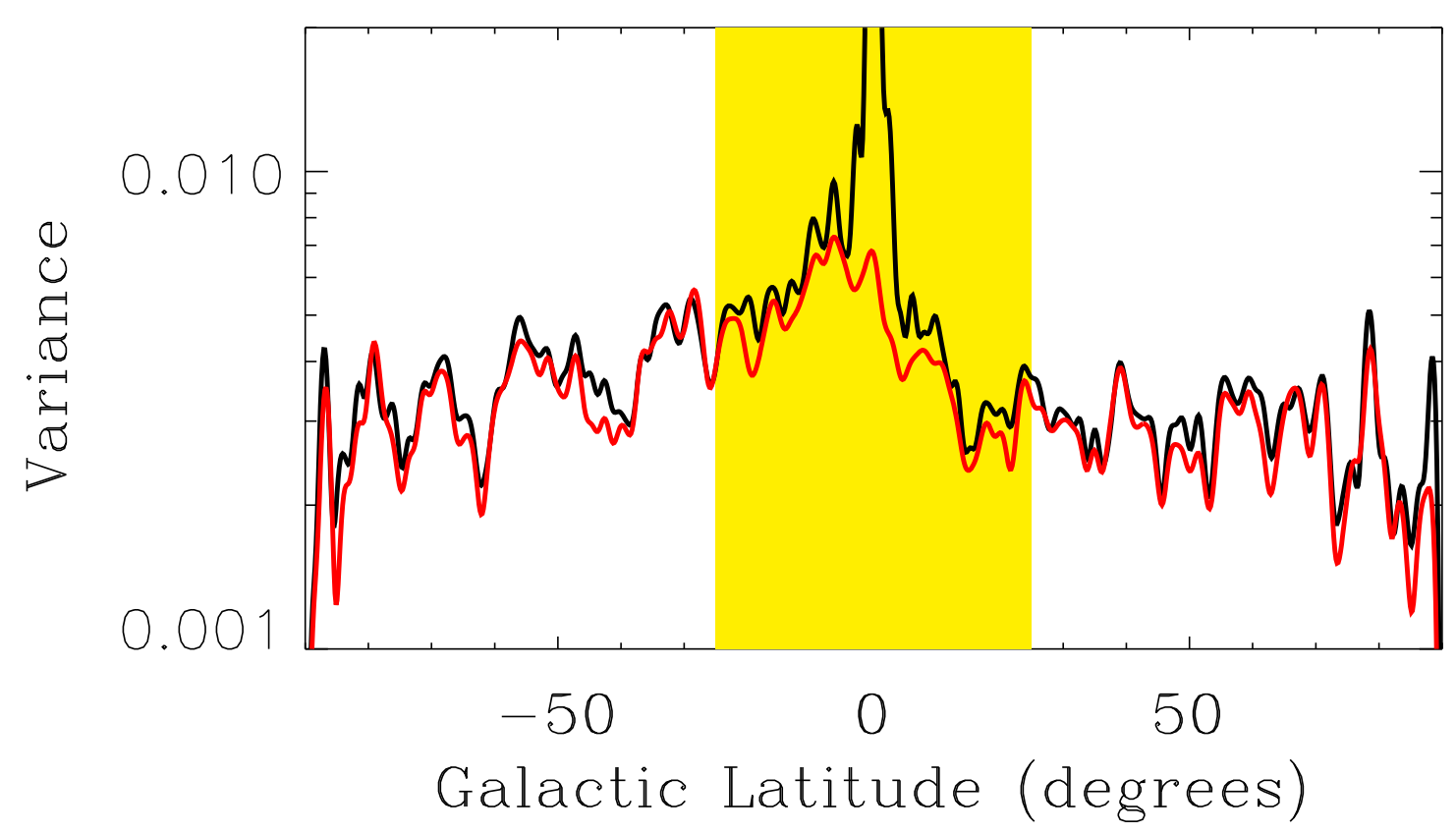

Figure 4. The variance for the WCM map (the black line) and the ILC III map (the red line) versus the galactic latitude.

the rings located at the North and the South hemispheres. To characterize this asymmetry we introduce the following parameter

$$
A(x=\cos \theta)=\frac{\operatorname{Var} T(-\cos \theta)-\operatorname{Var} T(\cos \theta)}{\operatorname{Var} T(\cos \theta)+\operatorname{Var} T(-\cos \theta)}
$$

where $\operatorname{Var} T(\cos \theta)$ is the variance of the signal for the ring with polar coordinate $\theta$.

In Fig.5 we show the behavior of the parameter of asymmetry $A(x)$ versus $\sin (b)$ in comparison with the same parameters taking from 1000 realizations of the RGF CMB. No additional analysis is required to observe that the variance of the Southern hemisphere is higher than for the North. This result is in agreement with investigations of the asymmetry of the power per $10^{\circ}$ patches of the sky [22], and analysis of peak statistics [23]. Furthermore, looking at Fig.4 at $b=-25^{\circ}$ one can find the global maxima of the variance outside the Kp0 mask. This zone is clearly seen in Fig.1 just below the galactic plane as a large cluster of minima mentioned by Park [27] Could the origin of this cluster be the same as for the cluster around the CS ? To answer this question we need to look closely on the properties of the signal for the ring $b=-57^{\circ}$ in the azimuthal direction. Note that this approach 


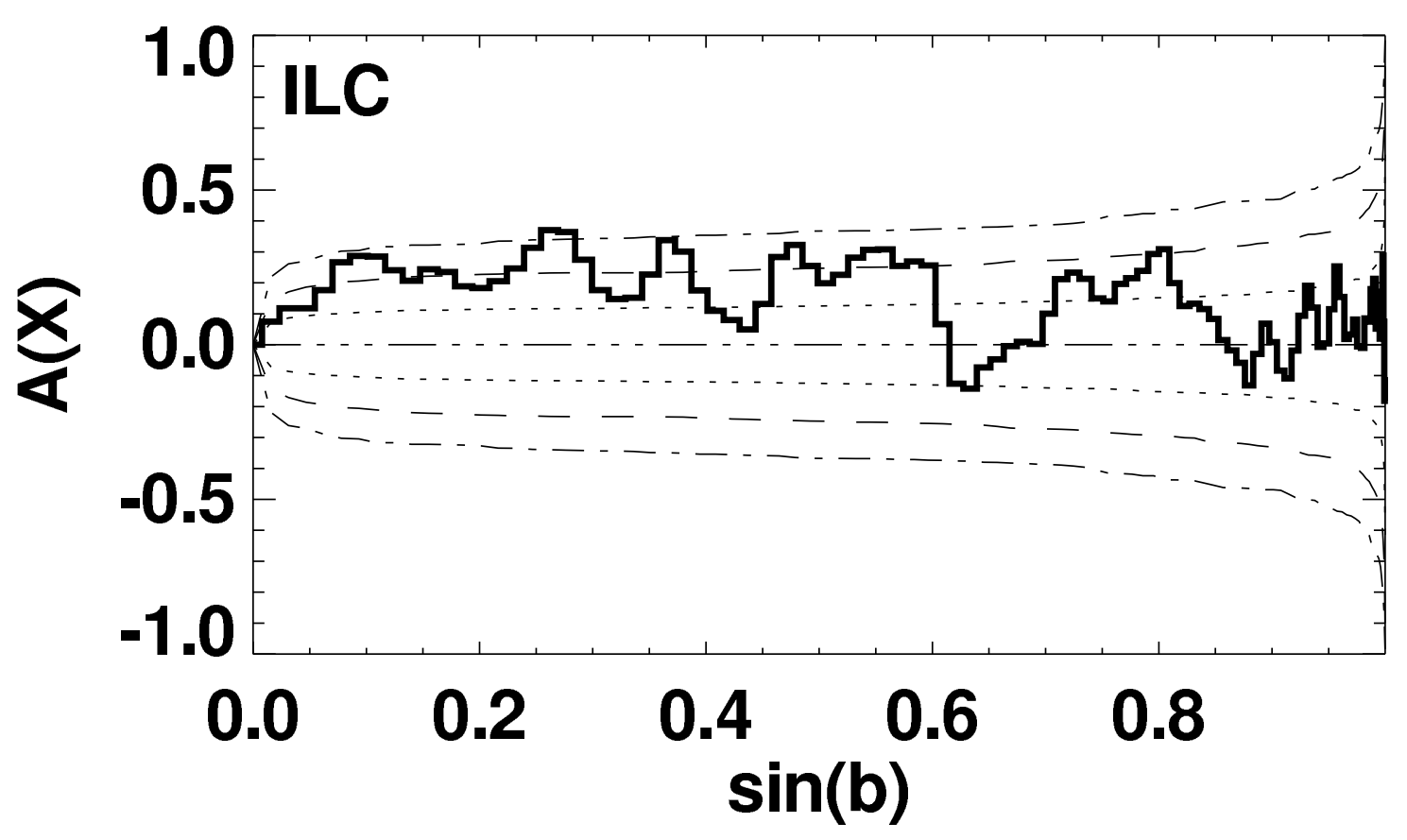

Figure 5. The asymmetry parameter $A(x)$ versus $\sin (b)$ for the ILC III rings and for 1000 realizations of the RGF CMB. The dotted, dashed and dash-dotted lines correspond to $68 \%, 95 \%$ and $99 \%$ threshoulds.

is close to the Eriksen et al. method [22], when the whole CMB sky was divided in zones with characteristic scales (diameter) about $10^{\circ}$, and after that for each zone the power of the signal was in used to characterize the difference between them. In our approach, we partially use the Eriksen et al. method, dividing the CMB sky in iso-latitude circles, and then by analysis of the morphology of the signal for each ring we will try to find a possible source of peculiarity of the signal. From Fig.5 clearly seen that the rings at $|\sin (b)|>0.82$ are characterized by the values of the parameter of asymmetry $A(x) \leq 0.2$. However, small values of this parameter tell us that the distribution of variance for each ring versus the galactic latitude is nearly symmetrical in respect to the Galactic plane. Thus, the CS detected for the $b=-57^{\circ}$, could have a "mirror partner" for the ring at $57^{\circ}$, or at least, the morphology of the signal for these rings could be close to each other to provide nearly the same variance $\operatorname{Var} T\left(b=-57^{\circ}\right) \simeq \operatorname{Var} T\left(b=57^{\circ}\right)$, and $A(x) \leq 0.2$. 


\subsection{The clusters in the ring at $b=-57^{\circ}$}

In this section we draw attention to the azimuthal distribution of the signal for the isolatitude ring with $b=-57^{\circ}$, which contains the CS. In Fig.6 we plot $T\left(\theta_{c}, \phi\right)$ for WCM and the ILC III maps smoothed by the angle $\Theta_{c}=1^{\circ}$. This figure clearly demonstrates that there are no significant differences of morphology of the CS in the ILC III and WCM maps Note that the WMAP team have pointed out that the ILCIII map is suitable for scientific analysis only for the range of multipole momentum $\ell \leq 10$. As it is seen from Fig.6 , for $b=-57^{\circ}$ ring there are no significant differences between the ILC III and WCM maps. This tendency is stable and for other rings outside the Kp0 mask. This is why below we use the name of the ILC III map as an indicator. All the analysis presented in the paper was performed for the WMAP ILC III maps in combination with the WCM map, and we did not find any significant differences between them.

In the following analysis we change the reference system of coordinate from the Galactic one to one in which $\phi=0$ is associated with the Galactic center and then all the values of $\phi$ are counted clockwise up to $\phi=360^{\circ}$. The GLESP pixelization allow us to fix the same number of pixels for each iso-latitude ring $(N=2048)$ and consequently the location of each pixel $0 \leq k \leq N$ is related to the angle $\phi_{k}$ as $\phi_{k}=2 \pi k / N$.

In Fig. 6 the CS is clearly seen at $k \simeq 1130 \div 1224$ as a cluster of minima $(\mathrm{C} 10, \mathrm{n}=10)$ of the signal in the WCM and ILC III maps. Moreover, there are no significant differences between these two signals either for the whole ring, or for the zone of the CS. Fig.7 shows the distribution of the length of the clusters versus the number of cluster. Clusters of maxima are shown in black, minima in red. As one can see from Fig.7 there are about 17 clusters of maxima and 17 clusters of minima. The mean length of the ILC and WCM clusters of maxima $\left\langle D_{\mathrm{ilc}}^{+}(n)\right\rangle \simeq 0.0337$, while for the clusters of minima we get $\left\langle D_{\mathrm{ilc}}^{-}(n)\right\rangle \simeq 0.0256$. For comparison, after taking 1000 realizations for the GRF with the WMAP best fit $\Lambda$ CDM power spectrum we have found $\left\langle D_{\text {grf }}^{+}(n)\right\rangle \simeq 0.0303$ and $\left\langle D_{\text {grf }}^{-}(n)\right\rangle \simeq 0.0315$. Remember that in the one-dimensional case the area statistic, used in [4] for two dimensional case and the length statistic, applied above are equivalent. Nevertheless, to estimate the probability distribution function $P(D)$ for the clusters at $\nu_{t}=0$ and to have a length $D$ within the interval $D-\delta D \div D+\delta D$ we performed the numerical simulations using 1000 realizations of the RGF CMB. In particular, for each of realization we took into account that the length of 


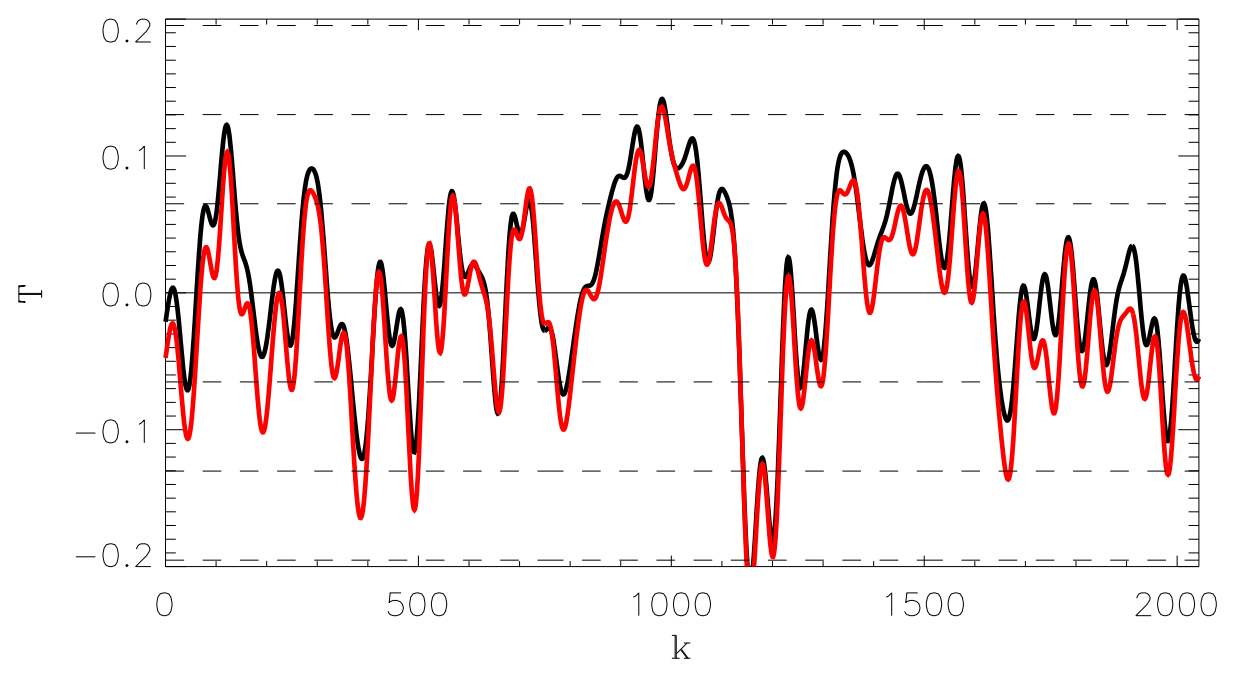

Figure 6. Plot of the temperature distribution in the one-dimensional ring through the center of the CS $\left(b=-57^{\circ}\right)$ in azimuthal direction. The solid line corresponds to the WCM signal and the red line is for the ILC III map. All the maps were smoothed by the angle $\Theta_{c}=1^{\circ}$. For this ring $\sigma_{0}=0.0651 \mathrm{mK}$. The dashed lines mark the thresholds $\left|\nu_{t}\right|=1,2,3$.

each ring with corresponding latitude $\theta$ is rescaled by factor $|\sin \theta|$, which re-normalize the mean length of the cluster for each ring. Then we got the number of the clusters with length $D$ versus its length and come to the distribution function $P(D)$. For $D>\langle D\rangle$ the function $P(D /\langle D\rangle)$ has Gaussian form $P(D) \propto \exp \left(-0.5(D /\langle D\rangle)^{2}\right)$ and corresponding probability to get the cluster with the length $D /\langle D\rangle \simeq 3-4$ is about $0.00033-0.011$. For the ring with $\theta=\pi / 2$ the total number of clusters is about 40 (for chosen resolution of the map $\Theta=1^{\circ}$ ), and the existence of a single cluster with $D /\langle D\rangle \sim 3$ is quite possible. However, the probability to get two or even three clusters with $D /\langle D\rangle>3$ for one ring is less then 0.016 ( for two clusters with $D /\langle D\rangle=3$ ), and less then 0.005 for three clusters with the same length. For $D /\langle D\rangle>4$ the corresponding probability to find two clusters is less then $2 \cdot 10^{-4}$. Note that our simulations are based on the RGF CMB map, which generally reproduced the statistically homogeneous and isotropic Gaussian random process. As it was pointed out in Section 3.1, the ILC III map is already statistically peculiar. Therefore, the model of the RGF CMB is not quite adequate to the WMAP ILC III or WCM maps, and corresponding estimation of the statistical properties of these signals needs to be taking into account as a tendency of departure from Gaussianity, rather then strong evidence of it. In Section 4 we 
will discuss some of the possible consequences of the anisotropy of the power, discussed in Section 3.1 and their influence on the properties of the zone with the CS.

Coming back to the analysis of the CS, we would like to mention that the cluster C10 containing the $\mathrm{CS}$ has length $D_{\mathrm{CS}}=0.0459 \simeq 1.5\left\langle D_{\text {grf }}^{-}(n)\right\rangle$ and its dimension is $D^{-}=2$ at $\nu_{t}=0$. At the same time $D_{\mathrm{CS}} /\left\langle D_{\mathrm{ilc}}^{-}(n)\right\rangle \simeq 1.8$ at $\nu_{t}=0$. These ratios tell us that this cluster looks like an "ordinary" cluster defined by the threshold $\nu_{t}=0$ without any significant deviation from the statistical properties of a random Gaussian Field. However, the extraordinary properties of this cluster become obvious when we take into consideration other thresholds, $-3 \leq \nu_{t} \leq-2$. From Fig. 7 we see that even for this range of $\nu_{t}$ the cluster still appears as a cluster of minima at the level $\nu_{t}=-3$, and local maxima at $\nu_{t}=-2$. Novikov and Jorgensen [21] have pointed out that for the cluster of minima with $d^{-}=2$ the conditional probability $P\left(k_{1}, k_{2}\right)$ to find two negative peaks located at the points $k_{1}$ and $k_{2}$ in this cluster is less than the conditional probability to find one maximum in between these points with height above $\nu_{t}$. In our case this threshold is $\nu_{t} \simeq-2$ and $P\left(k_{1}, k_{2}\right) \leq 0.02$. Note that the conditional probability $P\left(k_{1}, k_{2}\right)$ had been found theoretically in [21] for a random Gaussian field. In practice to prove this expectation we took under consideration 1000 realizations of the whole sky CMB maps from the random Gaussian generator and fix only those of the points of extrema (minima and maxima) which exceed the threshold $2 \sigma$. Then, for these points of extrema, we select the only those which have a structure of a cluster with two maxima and the single minimum between them (or two minima and the local maximum between them) with the amplitude of the corresponding minimum (or maximum) $\nu_{m} \geq 2 \sigma$, where $\sigma^{2}$ is the variance of the CMB signal for each realization of the CMB sky. The angular resolution of the RGF CMB maps corresponds to the $1^{\circ}$ and the power spectrum of the CMB $C(\ell)$ used for generation of the ensemble of realizations corresponds to the WMAP best fit $\Lambda \mathrm{CDM}$ cosmological model [28]. We have found that 38 (17 minima and 21 maxima) realizations from 1000 reveal similar to the CS morphology for particular isolated zones. However, none of those 38 realization were surrounded by the positive (or negative) clusters with dimension 4 or 5 . After detection of the isolated zones similar in morphology to the CS, we extracted one-dimensional cross-sections of the map through these zones in order to compare the properties of the one dimensional scan and the $b=-57^{\circ}$ ring from the ILC III map. Neither instrumental noise nor the beam profile were taken into account. Our motivation for using this simplest model is that the characteristic 
scale of the CS, mentioned in $[2,4]$ is about $10^{\circ}$. For these angular scales the contribution of the instrumental noise and the antenna beam shape are too small to affect the properties of the signal in the vicinity of the CS. Moreover, the structure of the CS is practically the same as for the WMAP first, third and fifth year data release, for which the instrumental noise is significantly different. We are planning to generalize our analysis, including mentioned above instrumental noise and beam shape in a separate paper.

Coming back to the analysis of the CS, we would like to point out that the CS cluster is not a unique feature of the $b=-57^{\circ}$ ring. There are two clusters of maxima (see Fig.6), namely the cluster C9 with $k_{\min }=825$ and $k_{\max }=1129$ with $D_{C 9}=0.15=4.40\left\langle D_{i l c}^{+}(n)\right\rangle$ and the cluster C11 with $k_{\min }=1310, k_{\max }=1634$, and $D_{C 11}=0.15=4.69\left\langle D_{i l c}^{+}(n)\right\rangle$. Here $k_{\min }, k_{\max }$ mark the coordinates of the cluster at $\nu_{t}=0$ in the pixel domain. These clusters seen in Fig.7 are near to the cluster of minima C10 within which the CS lies.

The existence of such clusters with lengths above $4\left\langle D_{\text {ilc }}^{+}(n)\right\rangle$ and the cluster of minima C10 with such large amplitude (negative) peaks seems to be a quite peculiar feature of the $b=-57^{\circ}$ ring. However, these peculiarities are not so specific. To show that "over-clustering" of the CMB sky is a typical feature of the signal let us examine, for example, the ring with $b=+57^{\circ}$, located symmetrically to the $b=-57^{\circ}$ ring in respect to the Galactic plane. In Fig.8 we show $T(k)$, and the distribution of clusters versus their length for the ring with $b=57^{\circ}$. This signal reveals a remarkable similarity of the morphology to the ring $b=-57^{\circ}$. As for the ring with CS, the ring $b=57^{\circ}$ is characterized by very high level of clusterization, the existence of the $3 \sigma$ minimum, as a member of the cluster with $D^{-}=4$, and the existence of the positive cluster with $D^{+}=6$. Moreover, one can see that the cluster of maxima C7 at $k_{\min }=547, k_{\max }=633$ has a structure similar to the structure of the signal in the CS, but now for the maxima.

At the end of this section we would like to point out that the existence of clusters with the length $D>3 \div 4\langle D\rangle$ is a quite rare event for the GRF. The presence of three clusters with $D \sim 3\langle D\rangle$ for single iso-latitude ring at $b=57^{\circ}$, and two clusters with $D>4\langle D\rangle$ for $b=-57^{\circ}$, as the CS as well, allow us to conclude that the ILC III and WCM maps are generally "over-clustered". Attention on the CS was focused mainly because of the amplitude of the signal in that position. A more specific feature of the CS is not this, but that it is really a cluster of peaks with nearly the same amplitude, and there is a very large cluster near to it. 

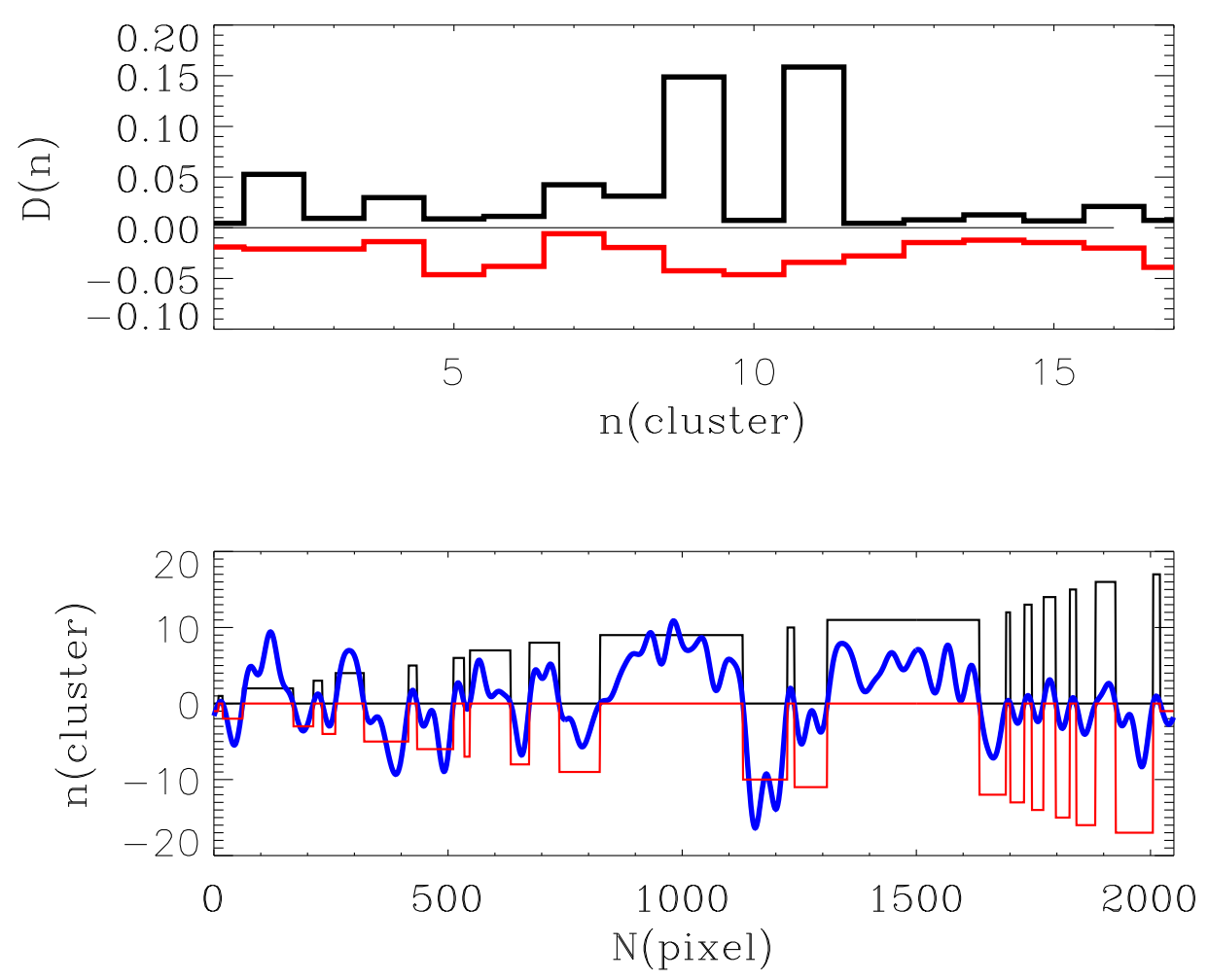

Figure 7. Top. The length $D(n)$ of the clusters $\nu_{t}=0$ versus the number of cluster. The positive line is for clusters of maxima, the negative line represents the minima. Bottom plot. Number of cluster versus its position in the pixel domain (2048 pixels correspond to the $360^{\circ}$ in azimuthal direction). The thick line corresponds to a one-dimensional ring of the ILC III map trough the CS in units of $5 * T_{k} / \sigma_{0}$. The negative sign for $D^{-}(n)$ marks the length of the clusters of minima.

\section{4. "DE-CLUSTERING" OF THE ILC III AND WCM BY LINEAR FILTRATION}

In this Section we re-examine an idea of Cruz et al. [4], that the origin of the CS can be explained if the WMAP CMB signal contains non-Gaussian components in combination with the Gaussian ones. This idea seems to be quite natural since the low multipole part of the WMAP ILC III map reveals significant peculiarities of the signal: alignment between quadrupole and octupole, coupling with the Galactic foregrounds, low power of the quadrupole, and so on. Note that the WMAP team performed the analysis of Gaussianity of the CMB signal by subtracting the ten multipole components of lowest order from the map and claimed that the rest of the signal is in agreement with Gaussian statistics. However, asymmetry of the power of the CMB, discovered [22] the existence of the CS and another 

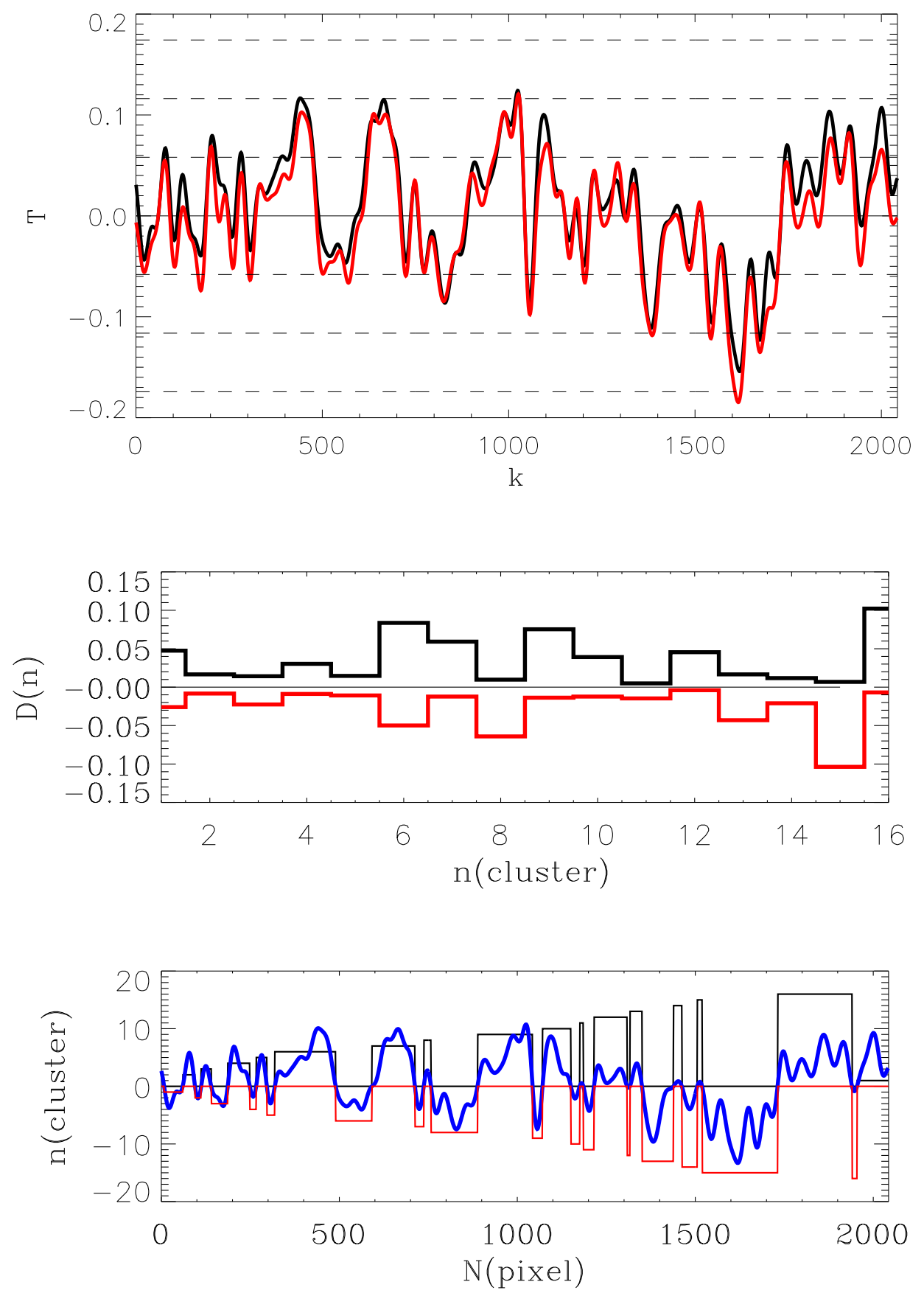

Figure 8. The same as for Fig.6 and Fig.7, but for the ring with $b=57^{\circ}$.

peculiarities, mentioned in $[30,31]$, raise the question of whether there is some mark imprint non-Gaussian features of the CMB, mainly localized in the low multipole range of the power spectrum.

If the WMAP CMB outside the Galactic mask $|b|=25^{\circ}$ is the sum of Gaussian and non- 
Gaussian components, it seems natural to guess that these two signals would have different characteristic scales. The idea, which we will develop below, is to use a linear filter of the CMB signal for each ring of the map with variable scale of filtration $R$, which can divide the CMB signal in two parts: $S(k)=\bar{S}(k, R)+s(k)$, where $s(k)$ corresponds to Gaussian component and $G(k)=\bar{S}(k, R)$ corresponds to non-Gaussian one. Note that Cruz et al. [? ] use the wavelet approach to perform this analysis. We will instead use the running window filter defined in the pixels domain as

$$
\begin{array}{r}
\bar{S}(k, R)=\left\langle T_{k}\right\rangle=\frac{1}{R} \sum_{j=0}^{R-1} T_{k+j-\frac{R}{2}}, \\
j=\left[\frac{R-1}{2}, . . N_{\text {pix }}-\frac{R+1}{2}\right] \\
s_{k}=T_{k}-\left\langle T_{k}(R)\right\rangle .
\end{array}
$$

Note that the choice of the linear filter is not so important for the criteria of separation of the Gaussian and non-Gaussian tails of the signal. One can for example use the Gaussian filter $\bar{S}(k) \propto \sum_{j} T_{j} \exp \left(-(k-j)^{2} / R^{2}\right)$, or any another reasonable filter. What is important is the chose of the criteria of the scale $R$. Following [9] we will use the skewness and kurtosis of the signals $s_{k}$ as a functions of $R$ trying to minimize the difference between their actual values and most probable values for the GRF.

In Fig.9 we show the skewness and kurtosis for the ILC III ring $b=-57^{\circ}$ for different scales of filtering $R$.

The skewness and kurtosis are very sensitive to the choice of the zone of the ring. For example, if we were to look at the zone $k_{\min }=825, k_{\max }=1634$ occupied by the clusters $C^{+} 9, C^{-} 10, C^{+} 11$ we get the skewness and kurtosis shown in Fig.9 (bottom plot). From these two plots one can see that the value of the parameter $R \simeq 70$ is preferred for this analysis. For this scale of filtering the kurtosis of the smoothed signal reaches a maximum, while for the Gaussian tail of the total signal both the characteristics are close to zero both for the whole ring and for the particular zone around the CS. In Fig.10 is shown the ILC III signal before (the red dotted line) and after subtraction of the smoothed over $R \simeq 70$ pixels (the black line). One sees that the CS is eliminated.

However, from the middle plot of Fig.10 we can see that the CS, as well as all the large clusters come from the smoothed signal, which preserves all the non-Gaussian features 

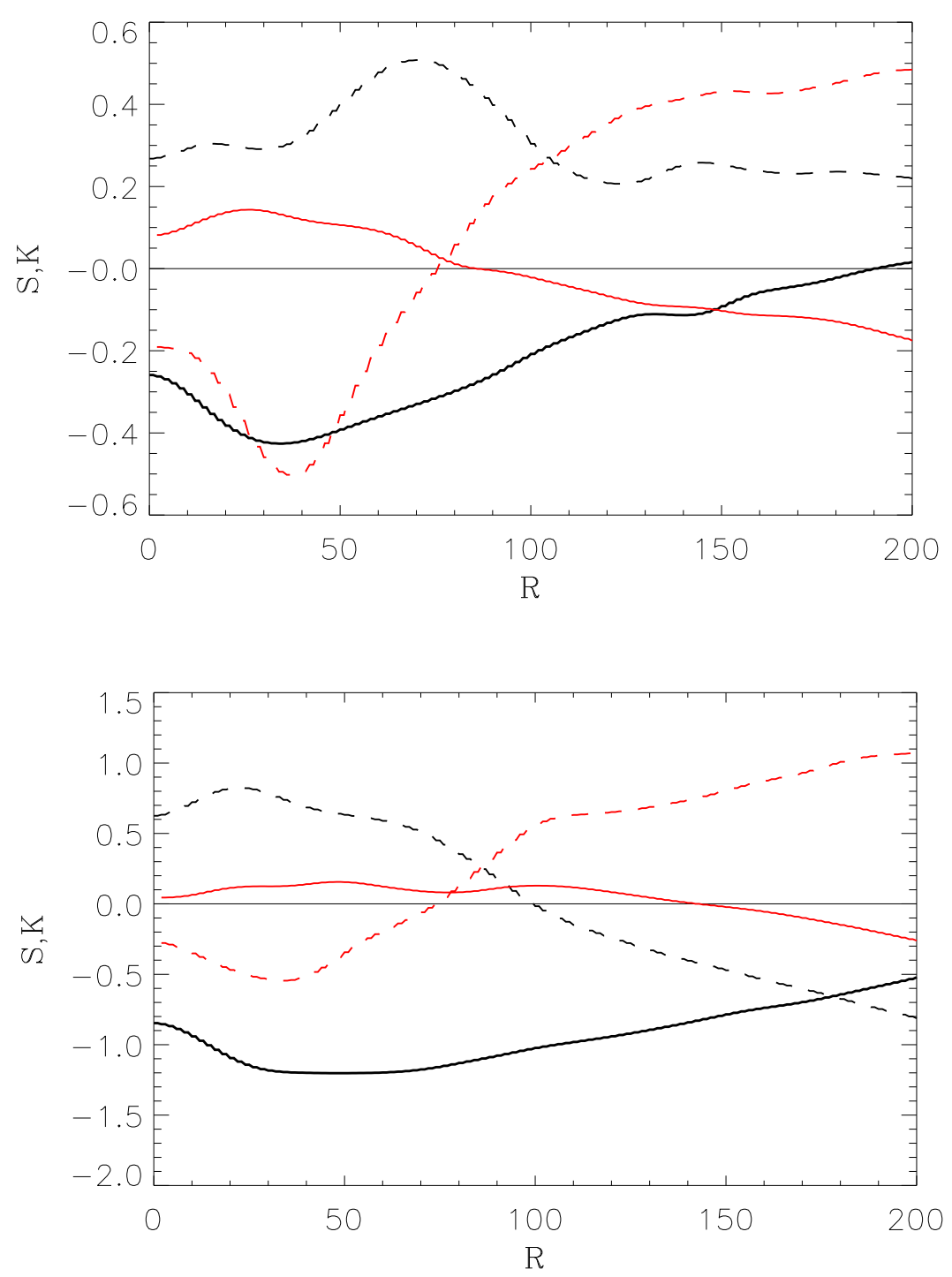

Figure 9. Top. The skewness and kurtosis of the ILC III $b=-57^{\circ}$ ring. The solid and the dash lines correspond to the skewness and kurtosis of the smoothed part of the ILC III signal $\bar{S}(k, R)$.

The grey lines are for the signal $s_{k}$. Bottom. The same as top, but for the zone of the ring

$$
k_{\text {min }}=825, k_{\text {max }}=1634 .
$$

mentioned in the previous section including the CS. The bottom plot of Fig.10 shows one more significant peculiarity of the CS zone. Just at the edge of the CS one can see a bright point source. The amplitude of this source is about $50 \sigma_{\mathrm{cmb}}$ for the $\mathrm{K}$ band and it drops down to $6 \sigma_{c m b}$ for $\mathrm{V}$ and $\mathrm{W}$ bands. In Fig.11 we show this point source for the Ka and $\mathrm{W}$ bands. For the ILC III and WCM maps the residuals from this point source are associated with local maximum at the level of $1 \sigma_{\mathrm{cmb}}$. 

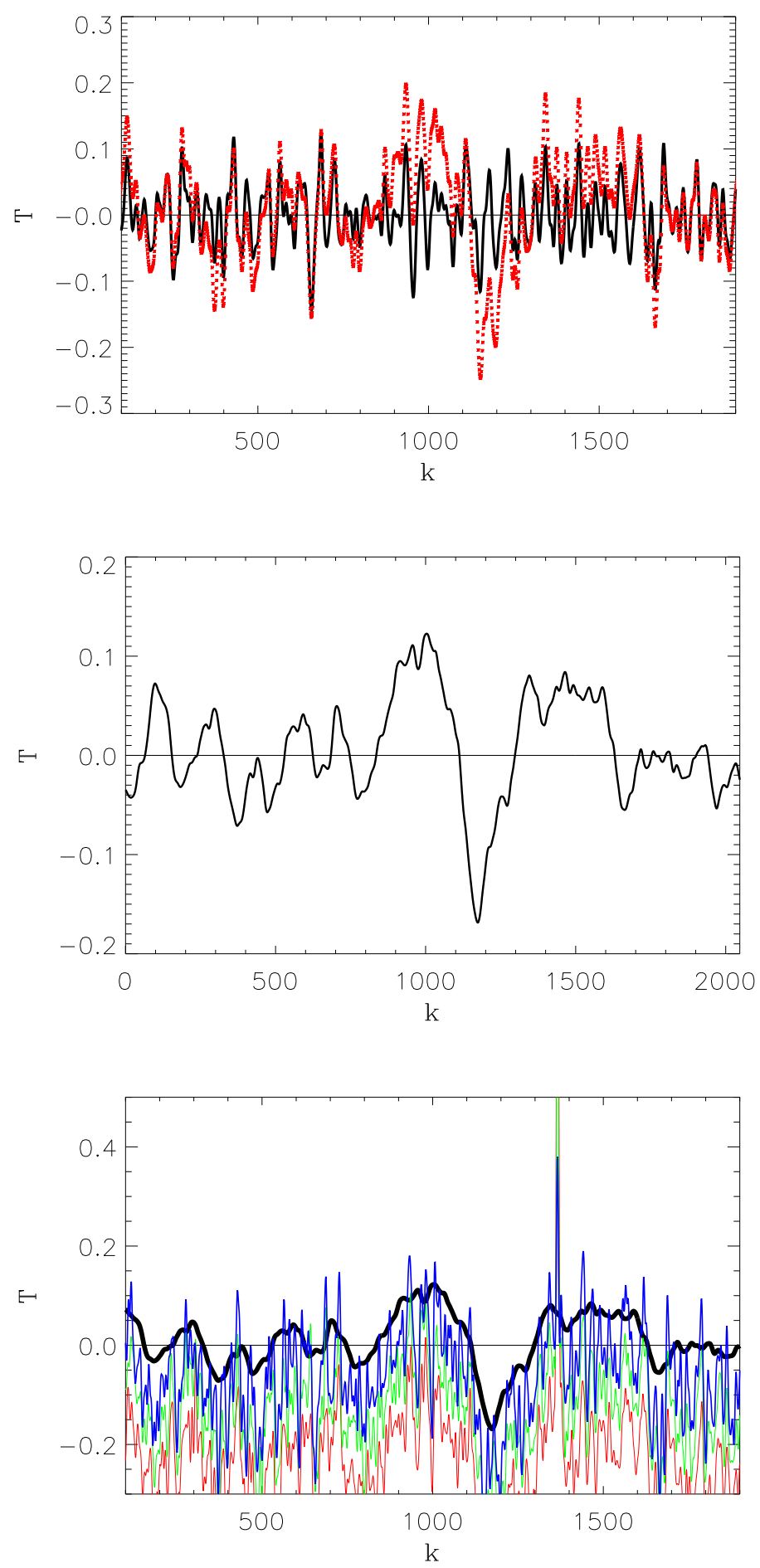

Figure 10. Top. The ILC III ring $b=-57^{\circ}$ before demodulation (grey dots) and after (black line). Middle. The ILC III signal, smoothed over $R=70$ pixels (the corresponding angular scale $\Delta \phi=12.3^{\circ}$.) Bottom. The smoothed ILC signal (thick black line) in comparison with signals from Ka, Q and V band. 

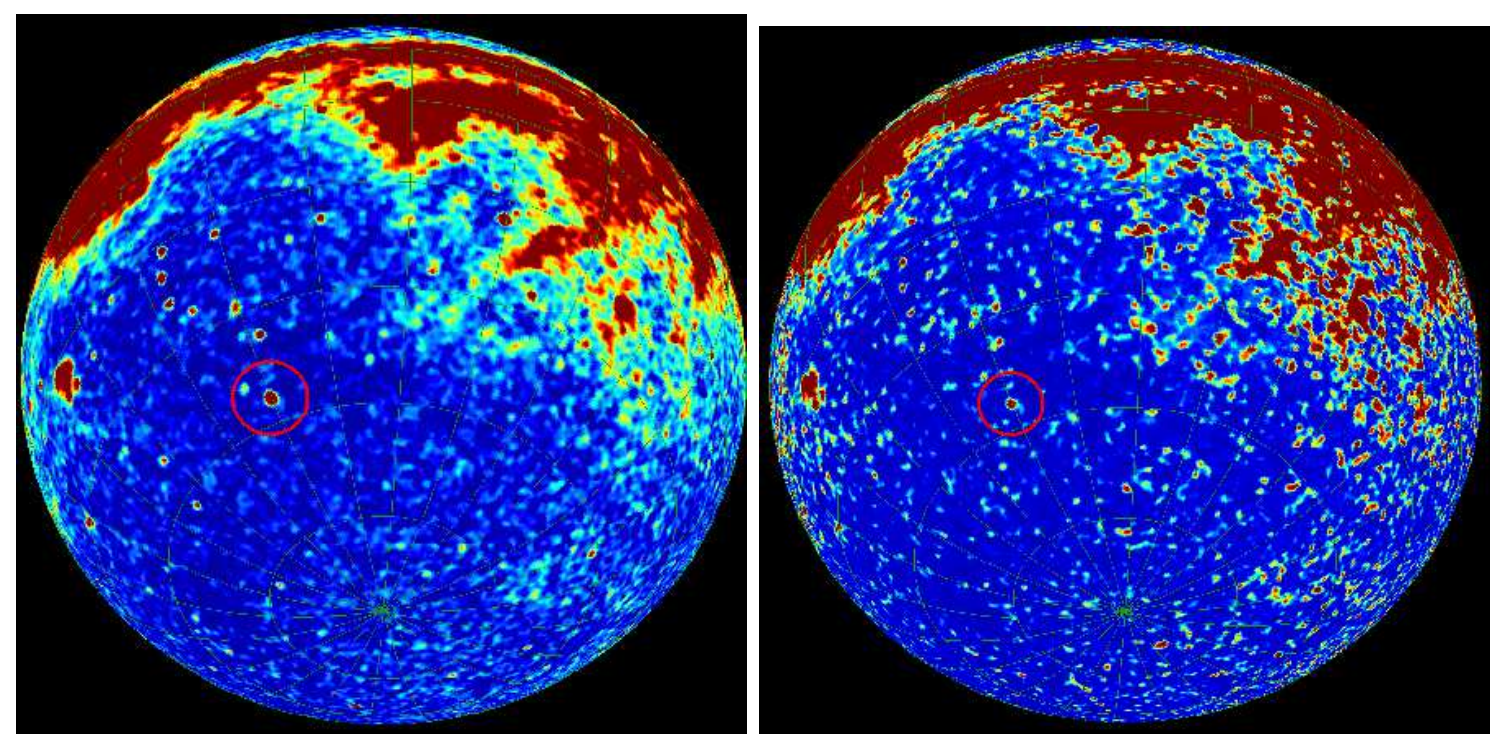

Figure 11. The Ka (left) and W (right) band signal from the South hemisphere. The red circle marks the location of the point source at the edge of the CS.

\section{ARE THERE OTHER "COLD" OR "HOT" SPOTS ?}

This question seems to be one of the most important question of the entire analysis of the origin of the CS. Do we have the only one single peculiar zone in the CMB map, or are there more zones with non-Gaussian properties of the signal ? To answer this question, we suggest using the distribution of the variance of the CMB signal versus the latitude, shown in Fig.5. Let us focus attention on the Southern hemisphere and in particular on the point of maximum of the variance. We can see the following coordinates of the points of maxima: $\theta=-80^{\circ},-70^{\circ},-57^{\circ},-30^{\circ}$. For these rings we performed the same analysis as for the ring at $b=-57^{\circ}$. All of them show the existence of non-Gaussian features, which can be identified by the same method as we have used for the $b=-57^{\circ}$ ring. In Fig. 12 we show the signal for the ring $-70^{\circ}$. A non-Gaussian hot spot clearly seen at $k \sim 850$ (the corresponding Galactic coordinates are $b=-70^{\circ}, \phi=149^{\circ}$ ). The amplitude of the peak is about $4.8 \sigma_{o}$, and it is the member of the cluster with $d^{+}=6$ for $\nu_{t}=0$. From Fig. 12 one can obtain the optimal size of the filter scale $R \simeq 50$.

Fig.13 shows the same characteristics of the signal as the previous one, but for the $b=$ $-30^{\circ}$ ring. Once again, the characteristic scale of filter is about $R=70$ pixels. One may continue the search for other rings belonging to the Southern hemisphere, just by following the distribution of maxima of the variance from Fig.5. However, the question is could we 
find the same peculiarities of the signal for the Northern hemisphere, where a deficit of the variance occurs? In Section 3 we already mentioned that the ring $b=57^{\circ}$ does indeed reveal over-clustering. Let us look closely at the skewness and kurtosis for that ring and find out the characteristic scale of non-Gaussianity. What is interesting is that for the $b=57^{\circ}$ ring the skewness and kurtosis shown in Fig. 14 are very close to the Gaussian characteristics, while for the zone $k_{\min }=1520, k_{\max }=1940$ with clusters $C^{-} 15, k_{\min }=1520, k_{\max }=1731$ and $C^{+} 16, k_{\min }=1732, k_{\max }=1940$ we can clearly see two minima for the demodulated signal, one at $R=20$ and another at $R=70$.

However, we stress that for the whole ring at $b=57^{\circ}$ even without filtering, the skewness and kurtosis are close to the GRF, unlike, for example $b=-57^{\circ}$ ring. Moreover, increasing the scale $R$ results in greater departures from Gaussianity, as it seen in Fig. 14. This tendency is common for $b=72^{\circ}$ ring (the point of local minimum of the variance), as for the $b=78^{\circ}$ (the point of local maximum of the variance from Fig. 5). Thus, significant non-Gaussianity of the ILC III and WCM maps mainly corresponds to the South hemisphere, and is associated with large angular scales, around $9-12^{\circ}$.

To show that clustering of the extrema of the ILC III and WCM signals is a typical feature of the morphology, we show in Fig.15 the ILC III map seen from the North and the South Galactic poles. From the ring with the latitude $b \simeq 80^{\circ}$ one can easily find a big cluster of maxima and one big cluster nearly at the same latitude. For the South pole the zone of the CS connects with three big clusters of maxima.

At the end of this section we would like to give one more argument that the over-clustering of the WCM and the ILC III maps can be related to the peculiar properties of the WMAP low angular resolution signal. In particular, we will show, that by changing the properties of the first 10 harmonic of the signal, we can easily destroy the non-Gaussianity of the CS. To show that we took under consideration the $a_{\ell m}$ coefficients of the ILC III map for $\ell \leq 10$ and replace them by the $a_{\ell m}^{\text {rand }}$ coefficients, taking from the random generator. The rest of the $a_{\ell m}$ for $11 \leq \ell \leq 100$ corresponds to the ILC III signal. If the non-Gaussianity of the CS is related to high multipoles, this replacement should not change significantly the statistical properties of the signal in the zone around the CS. However, if the non-Gaussianity of the CS is related to the correlations between low multipoles $(\ell \leq 10)$, the peculiarity of the CS should be broken. In Fig.16 we plot the ILC III map for $\ell \leq 10$, the RGF CMB map with the same resolution and the combined map in which the first 10 the ILC III coefficients $a_{\ell m}$ 
were replaced by the coefficients $a_{\ell m}^{\text {rand }}$.

One can see, that the CS zone is still visible in the combined map, but the amplitude and the morphology of the CS are changed dramatically. Firstly, the CS is no longer one of the deepest minima in the map. The amplitude of the CS drops down to $-0.15 \mathrm{mK}$ from $-0.22 \mathrm{mK}$. Secondly, the morphology of the zone around the CS is no longer corresponds to the cluster with dimension 2 , surrounded by the clusters with dimension 5 . The morphology of this zone now is close to the morphology of the signal shown in Fig.10 (top panel, the black solid line). This result is not surprising at all. As it was shown by [29] the CMB signal can be represented as a set of peaks with different amplitudes. For the RGF CMB signal the number density of these peaks and the shape of each peak in the vicinity of maxima depends on spectral parameters $\sigma_{0}, \sigma_{1}$ and $\sigma_{2}$, where

$$
\begin{gathered}
\sigma_{0}^{2} \sim \frac{1}{4 \pi} \sum_{m}(2 \ell+1) C(l), \quad \sigma_{1}^{2} \sim \frac{1}{4 \pi} \sum_{m}(2 \ell+1) \ell(\ell+1) C(l), \\
\sigma_{2}^{2} \sim \frac{1}{4 \pi} \sum_{m}(2 \ell+1) \ell^{4} C(l), \quad R_{1}=\frac{\sigma_{1}}{\sigma_{2}}, \quad \gamma=\frac{\sigma_{1}^{2}}{\sigma_{0} \sigma_{2}},
\end{gathered}
$$

where $C(\ell)$ is the power spectrum of the RGF CMB. It is well known that the parameter $R_{1}$ determines the correlation length of the CMB signal, which is proportional to the length of the cluster with dimension $1\left(\nu_{t}=0, \nu=1\right)$. As it is seen from Eq.(16, for $C(\ell) \propto$ $1 / \ell(\ell+1)$, this parameter mainly depends on the maximal resolution of the map. However, the clustering of the peaks is determined by the parameter $\gamma$ [21] This parameter depends on the variance $\sigma_{0}$, which for $C(\ell) \propto 1 / \ell(\ell+1)$ has logarithmic behavior. Thus, the value of $\sigma_{0}$ depends on the low multipole part of the power spectrum and consequently, the $\gamma$ parameter reflects directly this tendency. Thus, replacing of the low multipole part of the ILC III signal by $a_{\ell m}^{\text {rand }}$ from the random Gaussian signal breaks down the asymmetry of the power spectrum of the combined ILC map.

Moreover, in the combined high resolution map the deepest negative peaks are related to the negative peaks, clearly seen in the RGF CMB low resolution map (the second from the top panel). This simplest method confirms our conclusion that peculiar structure of the cluster around the CS is determined by the low multipole part of the ILC III signal, which reveals significant peculiarities. 


\section{COMPARISON WITH PECULIAR ZONES DETECTED BY WAVELETS}

Detection of peculiarities in the CMB sky is obviously one of the major steps in the investigation of the departure of the signal from statistical isotropy and homogeneity. As was mentioned in the introduction, this problem was discussed in [30] where Spherical Mexican Hat Wavelet (SMWH), elliptical SMHW, and Spherical Butterfly Wavelet (SBW) approaches were applied to the detection and location of the position of the spots in the CMB sky correlated with NVSS point sources catalog. Below we refer to these methods collectively as wavelet methods. McEwen et al. [30] summarize the detection of peculiar zones with approximate (estimated) coordinates of these zones. We present these data in Table 1 in order to compare these results with our results of detection of the high dimensional clusters

Let us discuss the properties of the signal for Zone 6 of Table1. The wavelet approach gives us the azimuthal coordinate of the spot $\phi_{6}=268^{\circ}$. From the top left plot, we can see that this point corresponds to a relatively small negative peak. The same morphology of the peaks is clearly observed at $\phi \sim 210^{\circ}$, and $\phi \sim 330^{\circ}$. Both peaks are very similar to the CS zone, but with smaller amplitudes. These two peaks probably contribute to the overall non-Gaussianity of the signal more significantly than the $\phi_{6}=268^{\circ}$ peak (see Table 1 ).

The next zone, which was not detected by cluster analysis at $\nu_{t}=0$, is Zone 7 shown on the top right plot. According to the wavelet analysis, the azimuthal coordinate for this zone is $\phi_{7}=213^{\circ}$. Once again, here we have a negative peak, but the peak at $\phi_{7} \simeq 240^{\circ}$ reveals a more significant departure from Gaussianity, it being a member of the cluster with number of minima $N=4$.

For Zone 8 , the wavelets analysis gives us $\phi_{8}=223^{\circ}$. We show the corresponding ring in Fig.17 (second from the top left plot). The detected zones manifest themselves as points of maxima, while the negative peaks detected by cluster analysis, listed in Table 1 reveal significant departure from Gaussianity. For example, the cluster of minima at $\phi \simeq 140-160^{\circ}$ can be detected not only for $\nu_{t}=0$ threshold, but even for $\nu_{t}=-2$, as a cluster of maxima and minima. Roughly speaking, all the pictures, shown in Fig.17 clearly demonstrate that implementation of cluster analysis with different thresholds $\nu_{t}$ allow us to detect not only single non-Gaussian zones, but also clusters of these zones which have non-local structure. To illustrate this tendency, we would like to draw attention to the bottom right picture belonging 
Table 1. The peculiar zones of the CMB sky detected by wavelets (first and second columns) from McEwen et al. (2006b). The second column shows the longitude $\phi$ and the latitude $\theta$ of each zone.

The third column shows the longitudes of the clusters where the wavelet peculiar point is the member. The forth column shows the number of extrema in the cluster $(S)$ and the length of the cluster $(D)$. The sign " $-"$ " means that the wavelet peculiar zone is not detected by CA with $\nu_{t}=0$.

Stars redirect the reader to Fig. 17.

\begin{tabular}{|c|c|c|c|}
\hline Zone & $\begin{array}{r}\text { Location, W } \\
\phi, \theta(\mathrm{deg})\end{array}$ & $\begin{array}{l}\text { Location, CA } \\
\phi_{\min }, \phi_{\max }\end{array}$ & $S, D$ \\
\hline 1 & 75,57 & $\begin{array}{c}56,86 \\
157,183 \\
267,304 \\
304,341\end{array}$ & $\begin{array}{ll}3, & 0.083 \\
3, & 0.072 \\
4, & 0.102 \\
4, & 0.102\end{array}$ \\
\hline 2 & 75,53 & $\begin{array}{r}69,93 \\
40,66 \\
160,183 \\
273,310 \\
\end{array}$ & $\begin{array}{ll}2, & 0.067 \\
4, & 0.072 \\
3, & 0.064 \\
4, & 0.103 \\
\end{array}$ \\
\hline 3 & 323,56 & $\begin{array}{l}304,339 \\
278,304 \\
\end{array}$ & $\begin{array}{ll}4, & 0.098 \\
3, & 0.072 \\
\end{array}$ \\
\hline 4 & 321,62 & $\begin{array}{l}306,333 \\
333,357 \\
272,307\end{array}$ & $\begin{array}{ll}2, & 0.075 \\
3, & 0.067 \\
4, & 0.097\end{array}$ \\
\hline 5 & 267,50 & $\begin{array}{c}251,274 \\
58,82 \\
313,344 \\
\end{array}$ & $\begin{array}{ll}3, & 0.064 \\
3, & 0.067 \\
4, & 0.086\end{array}$ \\
\hline 6 & 268,45 & $\begin{array}{c}- \\
129,156 \\
\end{array}$ & $\begin{array}{c}- \\
3,0.075 \\
\end{array}$ \\
\hline 7 & 213,40 & $\begin{array}{c}- \\
30,58 \\
233,258\end{array}$ & $\begin{array}{c}- \\
4,0.078 \\
4,0.069\end{array}$ \\
\hline 8 & 223,30 & $\begin{array}{c}- \\
9,37 \\
136,157\end{array}$ & $\begin{array}{c}- \\
4,0.078 \\
4,0.056\end{array}$ \\
\hline 9 & 160,26 & $\begin{array}{c}- \\
174,213 \\
4,32\end{array}$ & $\begin{array}{c}- \\
6,0.108 \\
4,0.078\end{array}$ \\
\hline 10 & $94,-28$ & $\begin{array}{c}- \\
35,62 \\
142,235 \\
333,352\end{array}$ & $\begin{array}{c}- \\
3,0.075 \\
15,0.258 \\
4,0.056\end{array}$ \\
\hline 11 & $81,-34$ & $\begin{array}{c}- \\
141,181 \\
187,230 \\
261,294\end{array}$ & $\begin{array}{c}- \\
7,0.11 \\
6,0.12 \\
7,0.092 \\
\end{array}$ \\
\hline 12 & $118,-42$ & $\begin{array}{c}- \\
8,30 \\
333,360+8\end{array}$ & $\begin{array}{cc}- \\
4, & 0.061 \\
5, & 0.097\end{array}$ \\
\hline 13 & $20,-48$ & $\begin{array}{c}7,30 \\
70,105 \\
243,278\end{array}$ & $\begin{array}{l}4,0.058 \\
4,0.097 \\
4,0.097\end{array}$ \\
\hline 14 & $34,-31$ & $\begin{array}{c}32,55 \\
288,330 \\
\end{array}$ & $\begin{array}{ll}5, & 0.064 \\
6, & 0.117 \\
\end{array}$ \\
\hline 15 & $230,-68$ & $\begin{array}{c}220,253 \\
19,52 \\
63,117\end{array}$ & $\begin{array}{cc}2, & 0.092 \\
2, & 0.092 \\
5, & 0.15\end{array}$ \\
\hline 16 & $204,-56$ & $\begin{array}{l}198,216 \\
148,188 \\
223,270\end{array}$ & $\begin{array}{cc}2, & 0.05^{*} \\
4, & 0.11 \\
5, & 0.13\end{array}$ \\
\hline 17 & $186,-54$ & $\begin{array}{l}193,216 \\
150,193 \\
224,294\end{array}$ & $\begin{array}{c}2,0.063^{*} \\
4,0.12 \\
6,0.194\end{array}$ \\
\hline & & 187230 & \\
\hline
\end{tabular}


to the Zone 18. According to Table 1, the non-Gaussian zone is located at $\phi_{18}=218^{\circ}$ being the member of the cluster and detected both by wavelet and by cluster analysis. However, there is another zone at $\phi \simeq 80^{\circ}$ with a morphology similar to the morphology of the CS. Moreover, looking at the shape of the signal at $\phi>130$ one can see the modulation of the signal by low frequency harmonics. This type of non-Gaussianity is an argument in favor of hypothesis that low multipoles of the CMB signal are highly non-Gaussian.

\section{CONCLUSION}

We have re-examined the properties of the Internal Linear combination WMAP CMB map and the co-added WCM map by an analysis of the properties of the signal in the vicinity of the CS. These two maps of the CMB signal display remarkably similar structures on equal latitude rings at $|b|>30^{\circ}$. We have re-examined the properties of the CS at the galactic latitude $b=-57^{\circ}$ and longitude $l=209^{\circ}$ and shown that it is associated with the cluster with length $D \sim 3\langle D(n)\rangle$. In addition to the CS, we have also found a few more zones of the CMB signal with almost the same morphology, at $b=57^{\circ}, b=-80^{\circ}, b=-30^{\circ}$.

From an analysis of the ILC III map we have shown that the shape of the CS is formed

primarily by the CMB signal localized in multipoles between $10 \leq \ell \leq 20$ (corresponding to angular scales about $5-10^{\circ}$ ), in agreement with results in $[4,9]$. At the same time we have demonstrated that the clustering of the peaks in the zone around the CS depends on the low multipole tail of the ILC III map $2 \leq \ell \leq 10$.

Taking into account that the same modes lead to a modulation of the whole CMB sky, we subtracted these modes from the CMB signal. The demodulated CMB signal looks like a random one without significant over-clustering in agreement with [2].

We have investigated the asymmetry of the variance for iso-latitude rings in respect to the Galactic plane. The South hemisphere has excess variance in comparison to the North hemisphere. This is why local defects and large clusters, including the CS and its associated cluster, are mainly concentrated in the Southern hemisphere.

Taking all these investigations together, we believe that the mystery of the WMAP CS directly reflects the peculiarities of the low-multipole tail of the CMB signal, rather than a single local (isolated) defect or a manifestation of a globally anisotropic model. This interpretation does not preclude the possibility of an exotic origin of the CS and related phenomena, 
but it does specify more precisely what properties such explanations must generate. A satisfactory model of the CS must explain the entire range of its behavior rather than only one aspect.

Our final remark is related to the definition of significance of the CS detection by different methods, based on the assumption that Gaussian statistics apply to the observed CMB sky. Ever since Eriksen et al. [22] showed that the distribution of the power of the CMB across the sky is very anisotropic at the scales about $10^{\circ}$, it has been clear that Gaussian statistics are no longer a valid reference for determining the significance of this feature. Our approach to the large-scale angular modulation of the CMB is a possible alternative approach to this issue.

\section{ACKNOWLEDGMENTS}

We acknowledge the use of the Legacy Archive for Microwave Background Data Analysis $\left(\mathrm{LAMBDA}^{2}\right)$. We also acknowledge the use of HEALPIX package [32] to produce $a_{\ell m}$. The GLESP package [20] was used in this work. OVV thanks Russian Foundation for Basic Researches by the grants No 09-07-00159, Foundation for the National Science Support and the "Dynasty" Foundation.

1. P. Vielva, E. Martinez-Gonzalez, R. B. Barreiro, et al., ApJ 609, 22 (2004), astro-ph/0310273.

2. M. Cruz, E. Martinez-Gonzales, P. Vielva and L. Cayon, MNRAS 356, 29 (2005), astro$\mathrm{ph} / 0405341$.

3. L. Cayon, J. Jin and A. Treaster, MNRAS 362, 826 (2005), astro-ph/0507246.

4. M. Cruz M., L. Cayon, E. Martinez-Gonzales, et al., ApJ 655, 11 (2007), astro-ph/0603859.

5. M. Cruz, E. Martinez-Gonzalez, P. Vielva, et al., MNRAS submitted, (2008), arXiv/0804.2904.

6. G. Hinshaw, D. N. Spergel, L. Verde, et al., ApJS 170, 288 (2007), astro-ph/0603451.

7. G. Hinshaw, J. L. Weiland, R. S. Hill, et al., ApJS 180, 225 (2009), arXiv:0803.0732.

8. D. N. Spergel et al., ApJS 170, 377 (2007), astro-ph/0603449.

\footnotetext{
${ }^{2}$ http://lambda.gsf c.nasa.gov
} 
9. M. Cruz, M. Tucci, E. Martinez-Gonzalez and P. Vielva, MNRAS 369, 57 (2006), astro$\mathrm{ph} / 0601427$.

10. K. Tomita, Phys. Rev. D 72, 103506 (2005), astro-ph/0509518.

11. K. T. Inoue and J. Silk, ApJ 664, 650 (2007), astro-ph/0612347.

12. J. J., Condon, W. D. Cotton, E. W. Greisen, et al., Astronom. J. 115, 1693 (1998).

13. L. Rudnick, S. Brown and L. R. Williams, ApJ 671, 40 (2007), arXiv:0704.0908.

14. T. Jaffe, ApJ 629, L1 (2005), astro-ph/0503213.

15. T. Jaffe et al., ApJ 644, 701 (2006), astro-ph/0512433.

16. L. Cayon et al., MNRAS 369, 598 (2006), astro-ph/0602023.

17. J. D. McEwen, M. P. Hobson, A. N. Lasenby and D. J. Mortlock, MNRAS 359, 1583 (2005), astro-ph/0406604.

18. J. D. McEwen, M. P. Hobson, A. N. Lasenby and D. J. Mortlock, MNRAS 371, L50 (2006), astro-ph/0604305.

19. M. Cruz et al., Science 318, 1612 (2007), arXiv:0710.5737.

20. A. G. Doroshkevich, P. D. Naselsky, O. V. Verkhodanov, et al., Int. J. Mod. Phys. D 14, 275 (2003), astro-ph/0305537.

21. D. I. Novikov and H. Jorgensen, ApJ 471, 521 (1996).

22. H. K. Eriksen et al., ApJ 605, 14 (2004), astro-ph/0307507.

23. D. L. Larson and B. D. Wandelt, ApJ 613, L85 (2004), astro-ph/0404037.

24. C. G. T. Haslam, C. J. Salter, H. Stoffel and W. E. Wilson, A \& A 47, 1 (1982).

25. J. M. Bardeen, J. R. Bond, N. Kaiser and A. S. Szalay, ApJ 304, 15 (1986).

26. L.-Y. Chiang and P. D. Naselsky, MNRAS 380, L71 (2007), arXiv:0705.1626.

27. C.-G. Park, MNRAS 349, 313 (2004).

28. E. Komatsu, J. Dunkley, M. R. Nolta et al., ApJS submitted, (2008), arXiv:0803.0547.

29. J. R. Bond and G. Efstathiou, MNRAS 226, 655 (1987).

30. J. D. McEwen et al., MNRAS 373, 1211 (2007), astro-ph/0602398.

31. J. D. McEwen, Y. Wiaux, M. P. Hobson, et al. MNRAS 384, 1289 (2008), arXiv:0704.0626.

32. K. Górski et al.ApJ 622, 759 (2005).

33. O. V. Verkhodanov, A. G. Doroshkevich, P. D. Naselsky et al., Bull. SAO, 58, 40 (2005). 

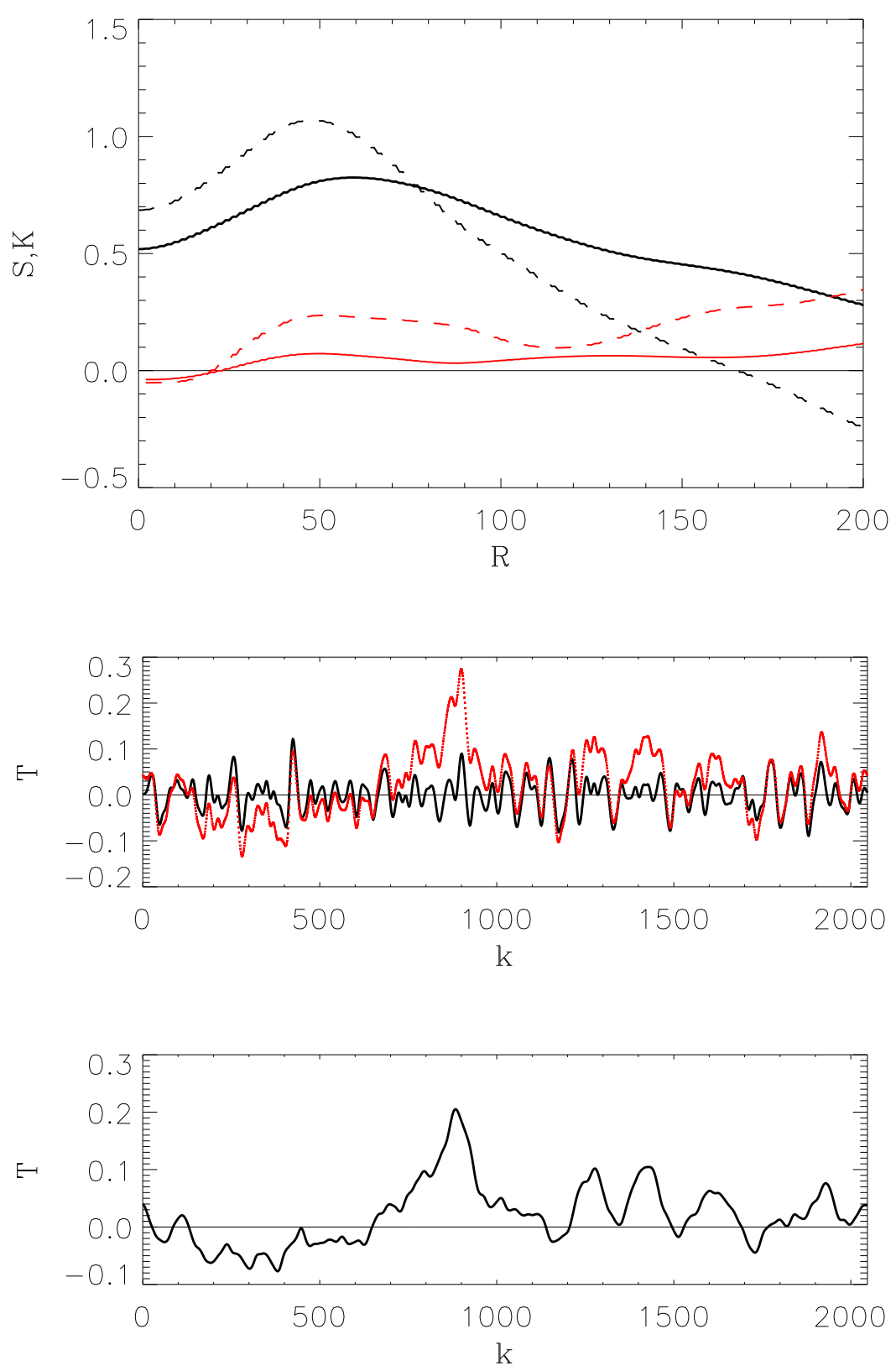

Figure 12. Top: The skewness and kurtosis for the whole $b=-70^{\circ}$ ring. The definition of the lines is the same as in Fig.10(top). Middle: The ILC III ring $b=-70^{\circ}$ before demodulation (the grey dots) and after (the black line). Bottom. The ILC III signal, smoothed over $R=50$ pixels (the corresponding angular scale $\Delta \phi=8.8^{\circ}$.) 

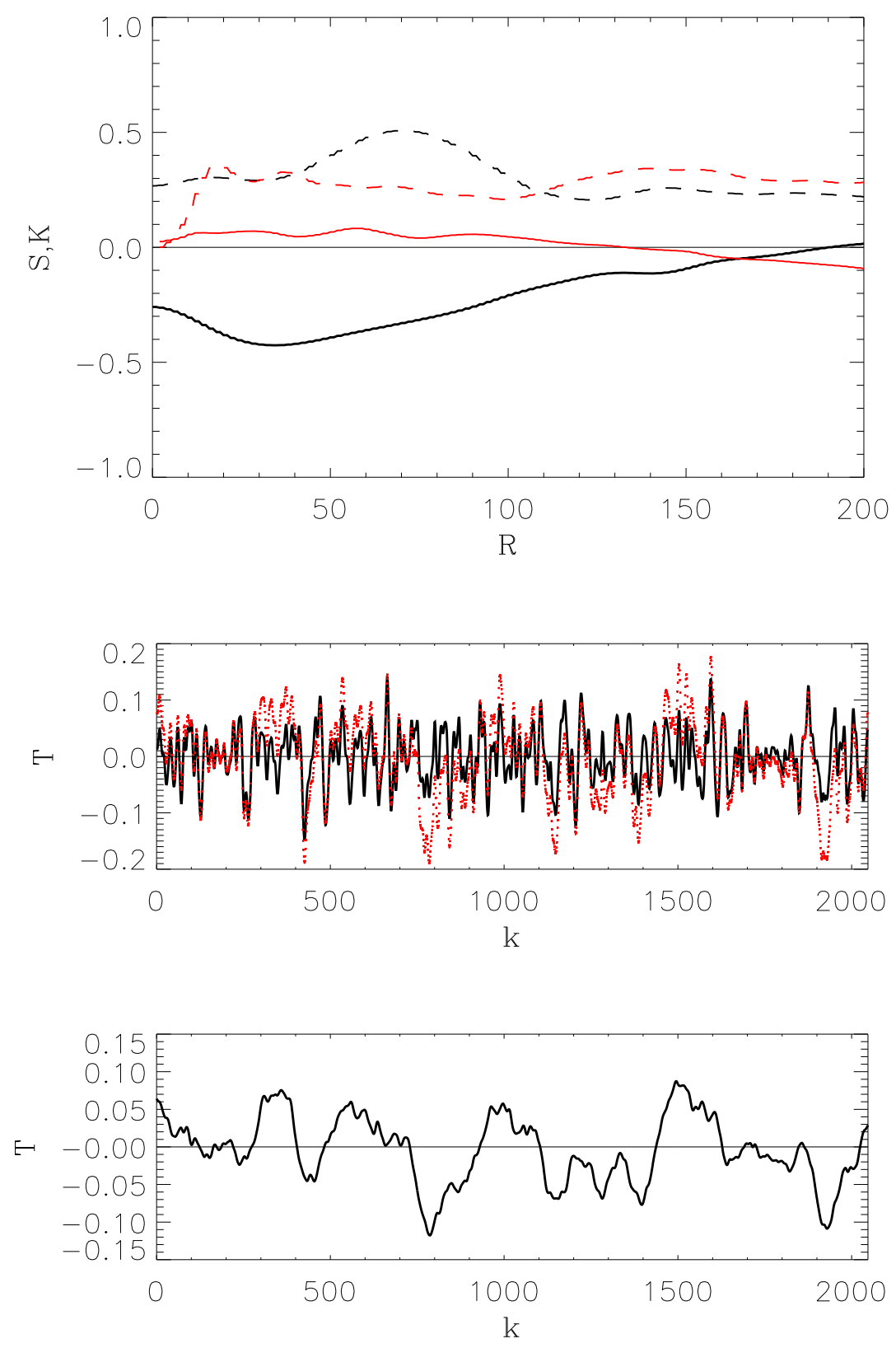

Figure 13. The same as Fig.10, but for $b=-30^{\circ}$ ring. The scale of filtration is $R=70$ pixels. 

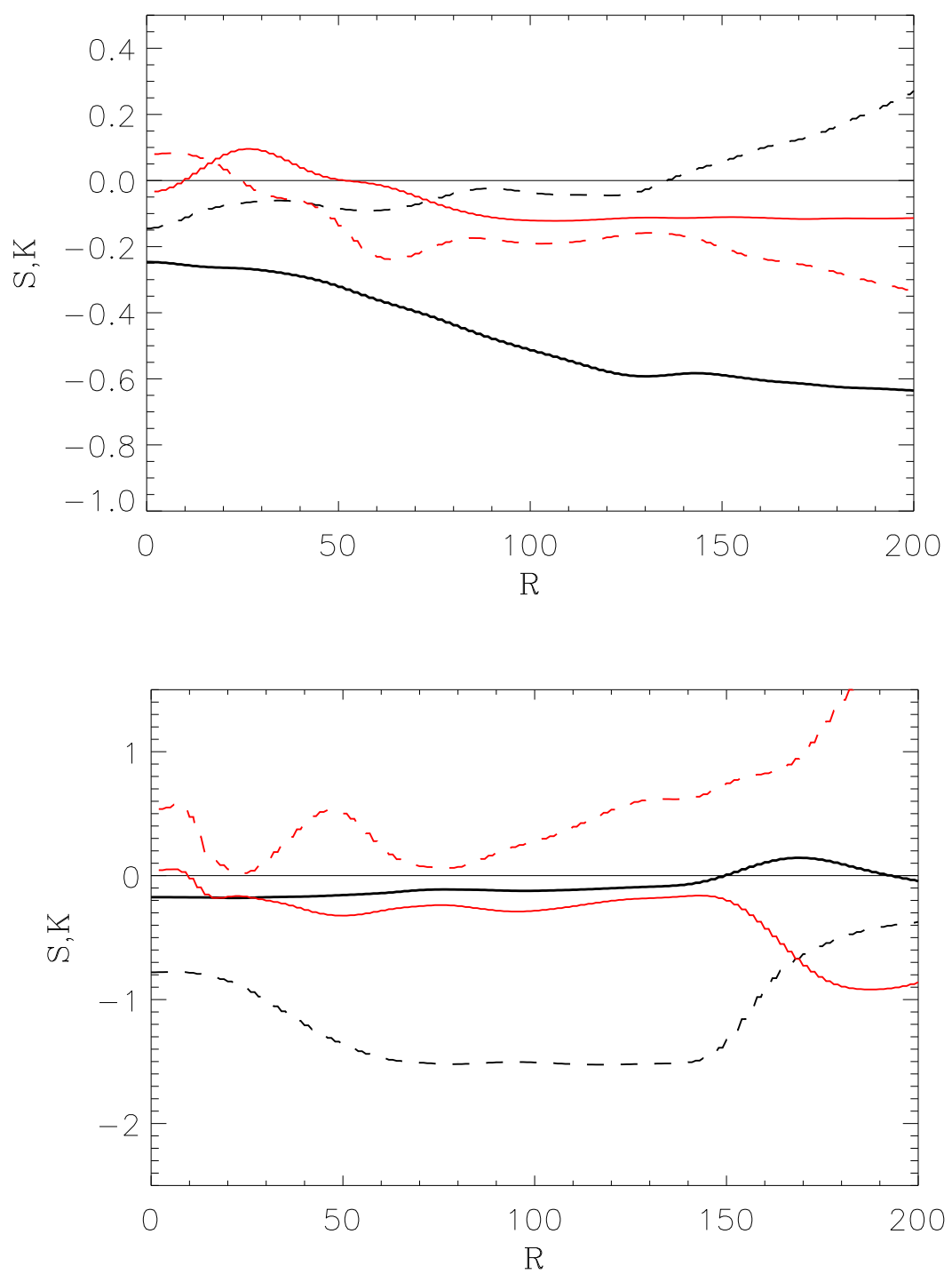

Figure 14. Skewness and kurtosis for the $b=57^{\circ}$ ring. Top plot show these characteristics for the whole ring, bottom plot is for the combination of the clusters $C^{-} 15+C^{+} 16$. The color of the lines is the same as for Fig. 9. 

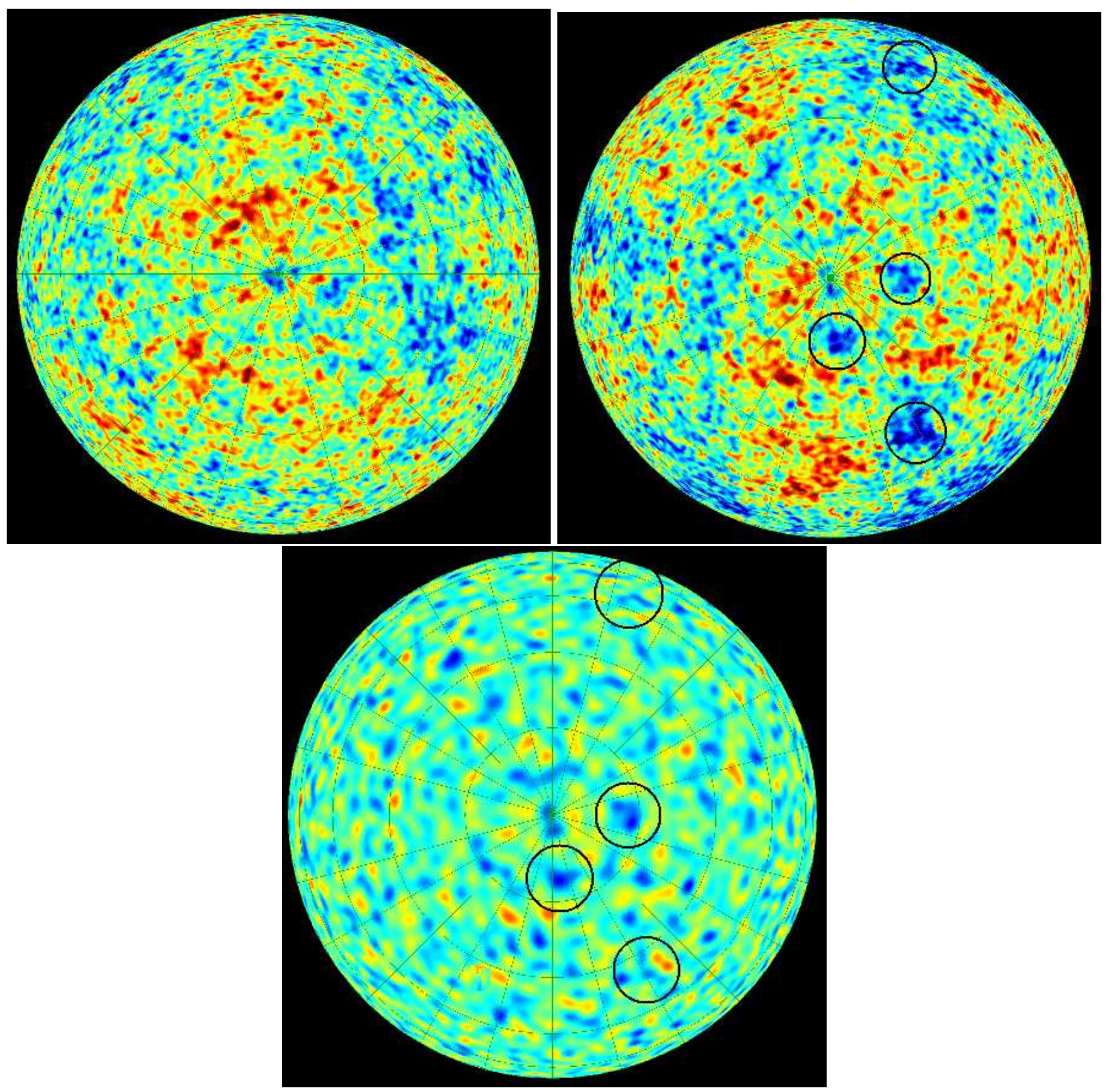

Figure 15. The image of the sky seen from the North and the South galactic poles (top and middle panels). The black circle marks the position of the CS. Bottom panel shows the South hemisphere of the CMB sky after subtraction of the first 20 multipoles of the ILC III signal. 

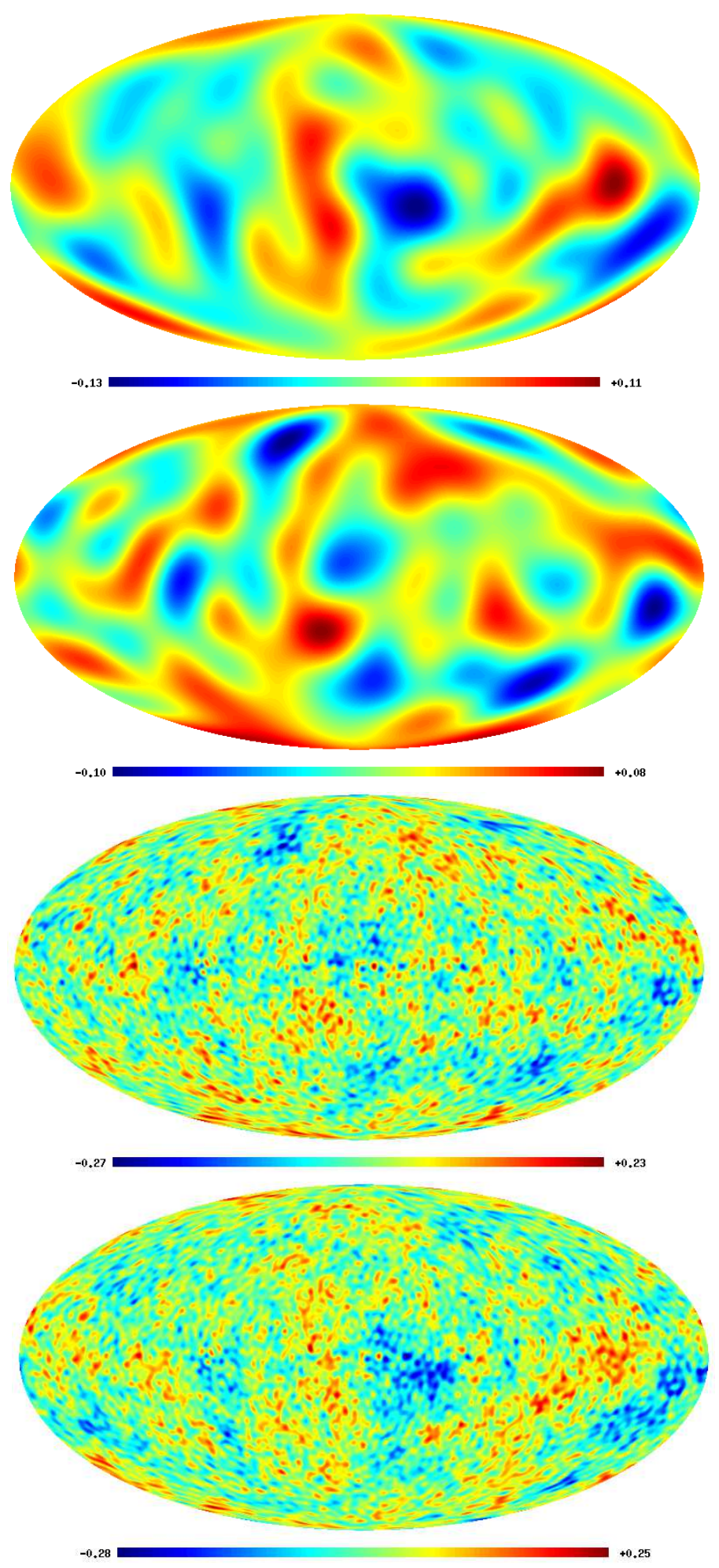

Figure 16. Top. The ILC III map for $\ell \leq 10$. The second one from the top is the map for RGF CMB with the same resolution as the top map. The second one from the bottom panel shows the combined ILC signal with first 10 multipoles taking from the random signal map (middle panel), and the rest of the multipoles $11 \leq \ell \leq 100$ from the ILC III map. The bottom map is the ILC III with $2 \leq \ell \leq 100$. 

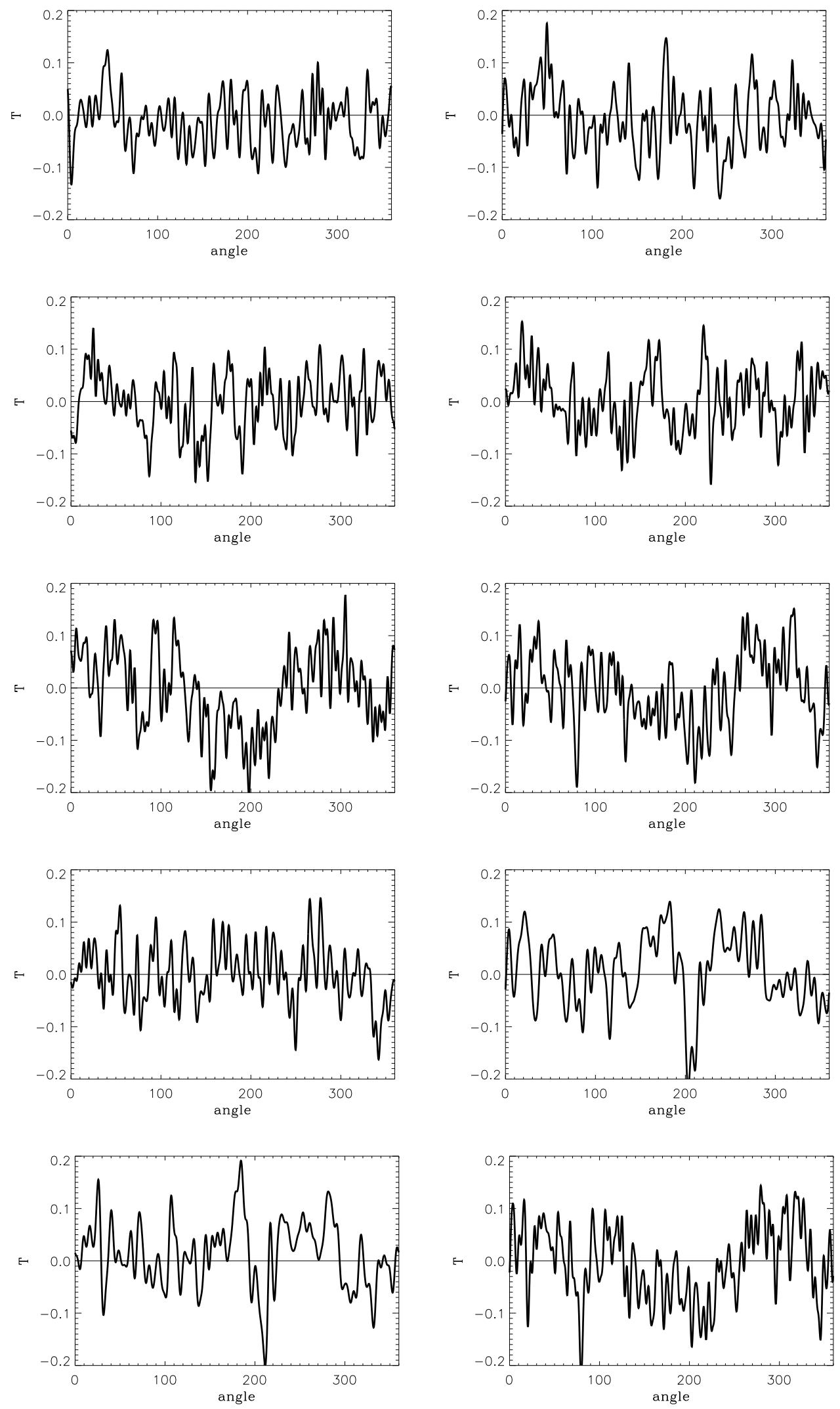

Figure 17. The CMB signal for equal latitude rings, marked in Table 1 by sign "-". From the top to the bottom and from the left to the right are the zones 6-12,16-18. 